\title{
What is the closest black hole to the Sun?
}

\author{
Cédric Foellmi*,a \\ ${ }^{a}$ Laboratoire d'Astrophysique de Grenoble, Observatoire de Grenoble, Université Joseph Fourier, CNRS UMR 5571, 414 Rue de la Piscine, \\ 38400 Saint-Martin d'Hères, France
}

\begin{abstract}
We examine the distance of the two galactic microquasars GRO J1655-40 and A0620-00 which are potentially the two closest black holes to the Sun. We aim to provide a picture as wide and complete as possible of the problem of measuring the distance of microquasars in our Galaxy. The purpose of this work is to fairly and critically review in great detail every distance method used for these two microquasars in order to show that the distances of probably all microquasars in our galaxy are much more uncertain than currently admitted. Moreover, we show that many confirmations of quantitative results are often entangled and rely on very uncertain measurements. We also present a new determination of the maximum distance of GRO J1655-40 using red clump giant stars, and show that it confirms our earlier result of a distance less than $2 \mathrm{kpc}$ instead of $3.2 \mathrm{kpc}$. Since it then becomes more likely that GRO J1655-40 could originate from the stellar cluster NGC 6242, located at $1.0 \mathrm{kpc}$, we review the distance estimations of A062000 , which is so far the closest black hole with an average distance of about $1.0 \mathrm{kpc}$. We show that the distance methods used for A0620-00 are also problematic. Finally, we present a new analysis of spectroscopic and astrometric archival data on this microquasar, and apply the maximum-distance method of Foellmi et al. (2006). It appears that A0620-00 could indeed be even closer to the Sun than currently estimated, and consequently would be the closest known black hole to the Sun.
\end{abstract}

\section{Introduction}

Microquasars are stellar binaries in which one of the component is a black hole. The stellar companion is filling its Roche lobe, ejecting matter through the first Lagrangian point, which then organizes itself around the compact object as an accretion disk. The temperature of the disk increases strongly towards the center and large amounts of X-rays with powerful, persistent and collimated jets are produced. In some cases, the relativistic jets appear to be superluminal. Microquasars are galactic laboratories of high-energy phenomena, and they must be seen as part of a large paradigm where AGNs, microquasars and possibly gamma-ray bursts (GRBs) share similar physics (Mirabel, 2004).

There are various physical reasons why the distance is an important parameter in our understanding of microquasars. Firstly, the superluminal velocity effect of

\footnotetext{
${ }^{\star}$ Based on data obtained at the European Southern Observatory (La Silla and Paranal Observatories), under program IDs 276.D5027(A), 066.D-0157(A), 70.D-0766(A) and 074.D-0204(A).

${ }^{*}$ Corresponding author

Email address: cedric.foellmi@obs.ujf-grenoble.fr (Cédric Foellmi)

Preprint submitted to Elsevier
}

the jets (which is a relativistic time delay effect producing an apparent velocity on the sky larger than the speed of light; see e.g. Rees, 1966) depends on the distance. Although it is a pure geometrical effect, it has a minimum threshold of $\beta=v / c \geq 2^{-1 / 2}$ (with $c$ the speed of light) below which the jets are not superluminal, whatever their true spatial speed $v$. When one tries to model the jets, and more importantly, the jet's launching mechanism, it is important to know the true velocity (and hence power) of the jets. Moreover, this effect is not very common, and it will become more important to correctly identify superluminal objects when the statistics will grow with future X-ray missions.

Secondly, the possible misalignment of the jets with respect to the accretion disk axis. As a matter of fact, GRO J1655-40 is often quoted as the typical microquasar where the jets and the disk are misaligned (e.g. Maccarone, 2002). This is directly related to the fact that the determination of the jet projection angle is linked to that of the distance, as explained below. The possible misalignment of the jet is an important clue of the formation and evolution of the system (see e.g. Martin et al. 2008).

Thirdly, the black holes' origin, and their orbit in November 7, 2018 
our Galaxy. This is particularly true for GRO J165540 , where a change in the distance by a factor of three makes a large difference to the orbit of the object in the Galaxy. See for instance Mirabel et al. (2002) who have calculated the orbit for GRO J1655-40 with $D=0.9$ and $3.2 \mathrm{kpc}$. This is important for our understanding of the origin of black holes in our Galaxy, and their distribution throughout the disk.

Finally, the true luminosity of the stellar companion can also be an important point in our understanding of the microquasars as dynamical objects, and in particular how the companion is affected by the filling of its Roche lobe and is certainly (partially) irradiated by Xrays from the accretion disk (e.g. Dubus et al. 1999).

The distance of microquasars is a simple yet central parameter in our description of these objects, conditioning a large part of our understanding both of the physics and the astronomical views of black hole stellar systems.

\subsection{The origin of the disagreement on the distance of GRO J1655-40}

The origin of this work is to be found in the publication by Mirabel et al. (2002) who note that the distance of GRO J1655-40 can be actually radically different from the accepted distance of $3.2 \mathrm{kpc}$ determined by Hjellming and Rupen (1995). This later value has since then been apparently confirmed by many other studies. In fact, GRO J1655-40 is a runaway black hole and Mirabel et al. published its proper motion, obtained with the Hubble Space Telescope. The opposite direction of the proper motion points almost perfectly (see their Fig. 1) to the center of a cluster (NGC 6242) located at $1.0 \mathrm{kpc}$ from the Sun (Kharchenko et al., 2005). It is then tempting to think that the system received a kick velocity at the moment of the primary's supernova explosion, and then moved away from its original cluster. This is the starting question: is the distance of GRO J1655-40 3.2 or $1.0 \mathrm{kpc}$ ? Among other things, the jets are superluminal with $D=3.2 \mathrm{kpc}$, but not at $1.0 \mathrm{kpc}$.

Foellmi et al. (2006) published a new method providing only an upper limit to the distance and giving $D \lesssim 1.7 \mathrm{kpc}$ when applied to GRO J1655-40, thus challenging the accepted distance of $3.2 \mathrm{kpc}$. This method is also partially problematic, and it will be discussed critically below. With a maximum distance of $1.7 \mathrm{kpc}$, GRO J1655-40 becomes a likely candidate of being the closest (stellar) black hole to the Sun. The position is currently held by A0620-00, which has an average measured distance of about $1.0 \mathrm{kpc}$. We will show however that the distance of A0620-00 is also very uncertain, revealing different problems in each distance method.

In summary, none of the published distances of GRO J1655-40 and A0620-00 are so far reliable. This paper emphasizes the difficulties encountered when determining the distance of microquasars, and shows that the distances of probably many, if not all, microquasars in our Galaxy are much more uncertain than currently admitted. In this paper we aim to provide a fair and critical review on the distance methods used on these two galactic microquasars. Other microquasars will be the subject of a subsequent work. In addition to Foellmi et al. (2006), partial material (incomplete and partially incorrect on the radio-jet distance of GRO J165540) has already been published in Foellmi (2006) and Foellmi (2007). This paper addresses many more important details, and in particular the issues of the dynamical studies used to determine the absolute magnitude of the secondary star of GRO J1655-40. Moreover, we also provide a completely new estimate of the distance of GRO J1655-40. Finally, we apply the method of Foellmi et al. (2006) to A0620-00, along with archival astrometric data. We conclude that GRO J1655-40 is certainly closer than the current accepted distance, but that A0620-00 might be even closer still.

\subsection{Distance methods: the basics}

The distance of a stellar object is often measured by comparing its absolute and apparent magnitudes. There are other methods, such as astrometry and parallax, and jet speed measurements (see below) for the special case of microquasars. But most often, the core method is simply this one. It reads:

$$
m_{\text {true }}-M=5 \log (D)-5
$$

where $m_{\text {true }}$ is the true apparent magnitude of the object, affected only by the geometrical distance separating the object and the observer.

There are (at least) two major issues with this method: one is general, and one is specific to stellar binaries of short period. The first observational difficulty is that $m_{\text {true }}$ is usually different from the observed apparent magnitude $m_{\mathrm{obs}}$ since the light is going through some absorbing patchy interstellar medium, which makes the star dimmer and redder. We have: $m_{\text {true }}=m_{\text {obs }}-A$, where $A$ is the absorption, in the given passband, expressed in magnitudes. Therefore:

$$
m_{\mathrm{obs}}-M-A=5 \log (D)-5
$$

The determination of $A$ is critical. It is often not measured directly, but rather is the so-called color excess, 
or reddening: $E(B-V)$, which is the relative amount of additional red color between the $B$ and $V$ bands due to the absorption of bluer wavelengths by the gas and the dust. For a star, the color excess can be obtained by comparing the observed and the intrinsic $B$ and $V$ color indices (the latter being inferred from the spectral type):

$$
E(B-V)=(B-V)_{0}-(B-V)
$$

The absorption in the $V$ band follows:

$$
A_{V}=R \times E(B-V)
$$

where $R$ is the total-to-selective absorption. For other colors, this relation needs corrections (see e.g. Fitzpatrick, 1999). The practice shows that what is often measured directly is $E(B-V), R$ being often approximated ${ }^{1}$ by a value between 3.0 and 3.7 with a moreor-less canonical value of 3.1. In fact, $R$ is a function of the interstellar reddening curve and the color of the stars, because the wide passbands of the photometric $B$ and $V$ filters makes the effective wavelengths of the filters shift with different stellar intensity gradients. Olson (1975) gives an approximate relation for $R$ :

$$
R=3.25+0.25(B-V)_{0}+0.05 E(B-V)
$$

where $(B-V)_{0}$ is the unreddened color index of the star. This relation is valid for normal stars with $(B-V)_{0}<0.4$ and $E(B-V)<1.5$ to within an error of 0.05 in $R(\mathrm{Ol}-$ son, 1975). See for instance Crawford and Mandwewala (1976) for a comparison for various photometric systems, and McCall (2004) for an updated discussion on this topic.

The other issue with the method consisting of comparing the magnitudes, specific to the stellar binaries with short period, is that the stellar companion (here the secondary star) is certainly not spherical anymore, since it completely fills its Roche lobe to feed the accretion disk. Moreover, its surface temperature might also not be homogeneous because of irradiation (e.g. Dubus et al., 1999, O'Brien et al., 2002), making its average observed temperature a function of the orbital phase. Hence uncertainties arise when one tries to estimate the true value of the absolute magnitude, $M$, in equation 2 since it is often calibrated with "normal" (spherical) and isolated stars.

The absolute magnitude is normally obtained through the determination of the spectral type of the star, which gives the temperature $T_{\text {eff }}$, and the modelling of either the radial-velocity curve or the multi-color lightcurves

\footnotetext{
${ }^{1}$ The difference between $R=3.0$ and 3.7 implies a relative distance uncertainty of 1.38 .
}

of the binary system gives the size of its orbit. From the latter, one can compute the effective Roche-lobe radius (see for instance Eggleton, 1983), which is then identified to the radius of the star. Assuming a uniform temperature distribution across the star and that it is roughly spherical, the luminosity (and thus the absolute magnitude) can be estimated with:

$$
L=4 \pi \sigma R^{2} T^{4}
$$

where $\sigma$ is the Stefan-Boltzmann constant.

\section{The published distance of GRO J1655-40}

GRO J1655-40 (a.k.a. Nova Sco 94) has been discovered as a Soft X-ray Transient (SXT) on July 27, 1994 with BATSE on board the Compton Gamma Ray Observatory (Zhang et al., 1994). Its jets were observed in the radio, giving a distance of about $3 \mathrm{kpc}$, implying that they are superluminal: $1.5 \pm 0.4 c$ (Tingay et al., 1995), $1.05 c$ (Hjellming and Rupen, 1995), where $c$ is the speed of light. GRO J1655-40 was the second superluminal source in our Galaxy shortly after the discovery of the jets in GRS $1915+105$ by Mirabel and Rodriguez (1994). The value of $3.2 \mathrm{kpc}$ has been published by Hjellming and Rupen (1995) based on VLA and VLBA radio data.

Since then, many publications use directly this canonical distance. For instance: Brandt et al. (1995); Barret et al. (1996); Regós et al. (1998); van der Hooft et al. (1998); Phillips et al. (1999); Shahbaz et al.(1999); Kuulkers et al. (2000); Soria et al. (2000); Greene et al. (2001); Combi et al. (2001); Buxton and Vennes (2001); Yamaoka et al. (2001); Gierliński et al. (2001); Kubota et al. (2001); O'Brien et al. (2002); Remillard et al. (2002); Kong et al. (2002); Kobayashi et al. (2003); Stevens et al. (2003); Willems et al. (2005); Brocksopp et al.(2006); Miller et al.(2006); Caballero-García et al. (2007); Martin et al. (2008) and Chakrabarti et al. (2008). More recently Shaposhnikov and Titarchuk (2009) use GRO J1655-40 and its parameters as a reference object for determining the mass of Cyg X-1. But a significant number of studies seem to confirm the canonical distance. We challenge here not only the lower limit of $3.0 \mathrm{kpc}$ of GRO J1655-40 but also every published confirmation.

We note however that Migliari et al. (2007) use with caution the upper limit of $1.7 \mathrm{kpc}$ determined in Foellmi et al. (2006).

\subsection{The radio-jet kinematic distance of $3.2 \mathrm{kpc}$}

Hjellming and Rupen (1995) present new radio data from which they infer a value for the distance of 
GRO J1655-40. The method is simple: the opposite motions of the receding and approaching jets $\left(\mu_{-}\right.$and $\mu_{+}$ respectively) are directly related to jet projection angle relative to the line of sight $\theta$, the true jet speed $\beta=v / c$, and the distance $D$. The authors measure the intrinsic proper motions of each jet: 54 mas d $^{-1}$ for the NE component, and 45 mas d $^{-1}$ for the SW component, and use the kinematic equation described in Mirabel and Rodriguez (1994):

$$
\mu_{ \pm}=\frac{\beta \sin (\theta)}{1 \pm \beta \cos (\theta)} \frac{c}{D}
$$

where $c$ is the speed of light. There are two equations and three unknowns. A constraint on the distance can be obtained by eliminating $\theta$ and requiring that $\beta<1$ :

$$
\frac{D}{c}\left(\frac{2 \mu_{+} \mu_{-}}{\mu_{+}+\mu_{-}}\right)=\frac{v}{c}<1
$$

Taking $v=c$, the maximum distance of GRO J165540 inferred from the proper motion of the radio jets is $D<3.5 \mathrm{kpc}$.

A constraint on the inclination angle of the jets can also be obtained by eliminating $v / D$ and requesting that $v<c$. We obtain:

$$
\frac{v}{c}=\frac{\left(\mu_{-}-\mu_{+}\right)}{\left(\mu_{-}+\mu_{+}\right)} \cos (\theta)^{-1}<1
$$

This gives: $\theta \leq 84.8^{\circ}$. Rearranging equation 7 and eliminating $\beta$, we can write, using the value of $\theta$ :

$$
\frac{1}{v / D}\left(\frac{2 \mu_{+} \mu_{-}}{\mu_{+}+\mu_{-}}\right)=\sin \theta \leq 0.996
$$

that is $v / D>49.3$ mas $^{-1}$. All these results are given in Hjellming and Rupen (1995).

At this point the authors mention that: "For a distance of $3.2 \mathrm{kpc}$, this corresponds to $v \geq 0.91 \mathrm{c}$, implying $84.3^{\circ} \leq \theta \leq 84.8^{\circ}$." Why $3.2 \mathrm{kpc}$ ? The only previous mention of the distance at the beginning of the article is only stating that the source lies "at a distance of about 3 kpc", and for which three references were given (all discussed below): McKay and Kesteven (1994); Harmon et al. (1995); Tingay et al. (1995). In fact, the value $D=3.2 \mathrm{kpc}$ is constrained to lie between the value of $3.0 \mathrm{kpc}$ considered as a lower limit and determined elsewhere, and the actual result on the upper limit of 3.5 $\mathrm{kpc}$. However, choosing $3.2 \mathrm{kpc}$ fixes $\theta$ that is now constrained to a very narrow range $\left(0.5^{\circ}\right)$, and the system is now defined.

\subsection{The confirmation of $3.2 \mathrm{kpc}$ with possible wiggles inferred from unaligned images}

Furthermore, this value of $3.2 \mathrm{kpc}$ is being confirmed by a model of the possible jet precession (adding two parameters to the kinematic model: the precession period and axis inclination). It is however barely relevant concerning the distance, since, as mentioned earlier, the systems characteristics are now fixed. This additional modelling gives $\theta=85^{\circ}$ based on the fact that the jet precesses, or more precisely: "[...] the jets 'wiggle' slightly about the best-fit position angle". The authors performed a detailed modelling, similar to what has been done by one of the authors on the galactic source SS433 which shows clearly jet precession (Hjellming and Johnston, 1981a b, 1988).

The model of the wiggles assume, of course, that they are true, and are due to the kinematics only. In Fig. 3. of Hjellming and Rupen (1995) we see the variations of position of roughly 4 different ejectas (following the main solid lines only). It is obvious that few points perfectly follow the best-fit constant proper motion. But it is not obvious at all that they form a regular periodic pattern. Succeeding at modelling these wiggles does not mean we can interpret them as the signature of jet precession taking place in GRO J1655-40, since the low number of points makes the fit poorly constrained. At that point, it should be clear enough that these supposed wiggles do not help to secure the distance. But there are additional problems attached to them.

In order to interpret wiggles as precession, one must ensure that the global motion follow lines of constant proper motion. But the 22 epochs of VLA observations used for by this model did not resolve the source at a level of 100 mas, but only as a multi-core object elongating with time. The reason why a constant proper motion is a reasonable hypothesis is because it is consistent with what is seen in the VLBA observations. But the authors emphasize the lack of a very-long-baseline interferometry calibrator, which implies that these VLBA data are self-calibrated, "eliminating all absolute positional information, and leaving the alignment of the different images a free parameter." Consequently, the fact that the brightest point in each image is the stationary center of ejection is an hypothesis.

Moreover, as mentioned in the paper, "these [VLA] and other [unspecified] data are consistent with constant intrinsic proper motion of 54 mas d $^{-1}$ " and later "the underlying proper motions appear constant". This value is in agreement with the result of equation 10 . The hypothesis of constant proper motion is strengthened by the fact that the daily Southern Hemisphere VLBI Experiment (SHEVE) array observations of Tingay et al. 
(1995) are consistent with the major structures of the $V L B A$ images of Hjellming \& Rupen. As a matter of fact, the authors note that the proper motion inferred from SHEVE data of $65 \pm 5$ mas $\mathrm{d}^{-1}$ actually agrees with the 62 mas $^{-1}$ motion of the outer edge of the early NE ejecta. All these measurements indeed appear to roughly agree, but the uncertainties are likely large enough to encompass the wiggles. In summary, not only are the mere existence of these wiggles doubtful, but the simple ability to extract meaningful and additional constraints from it is also questionable.

We conclude that the value of $3.2 \mathrm{kpc}$ has been chosen between a firm upper limit of $3.5 \mathrm{kpc}$ and the external indication that its lower limit must be "about $3 \mathrm{kpc}$ ", which consequently fix the value of $\theta$. A questionable model is used to strengthen its value, and, as an obvious consequence, confirm the distance value. Literally, later in the text, it is said that the "[...] kinematic model for GRO J1655-40 gives a distance of $3.2 \pm 0.2 \mathrm{kpc} "$.

\subsection{Where does 3 kpc come from?}

As noted above, three references are given for a first estimation of the distance: Harmon et al. (1995), Tingay et al. (1995) and McKay and Kesteven (1994). Unfortunately, the first reference is literally citing the two others for the distance value, and must therefore be discarded. The paper by McKay and Kesteven (1994) is actually an IAU Circular which simply states that "HI observations of GRO J1655-40 made with the AT Compact Array show solid absorption in the velocity range +10 to $-30 \mathrm{~km} \mathrm{~s}^{-1}$, with a further isolated weak feature at $-50 \mathrm{~km} \mathrm{~s}^{-1}$. The balance of probabilities is that the distance is around $3.5 \mathrm{kpc}$, unless the $-50 \mathrm{~km} \mathrm{~s}^{-1} \mathrm{fea}$ ture is due to an atypical cloud." Although not being a robust measurement, the result obtained in this Circular needs to be verified.

Identifying the origin of the negative-velocity features in the absorption spectrum is the crucial point, since it provides an estimate of the lower limit of the distance, if correctly interpreted (i.e. if correctly identified and attributed to a component whose velocity can be estimated). To interpret the radio spectrum, one must consider a background source of unknown distance emitting continuum radiation (here GRO J1655-40) that is being intercepted by foreground $\mathrm{HI}$ clouds. When one looks inside the solar galactic orbit, the line of sight goes through the multiple galactic spiral arms. Assuming all the clouds are moving with the mean galactic rotation scheme, the more distant from the Sun the cloud is, the more negative is its velocity, in the Local Standard of Rest, down to the tangential point where the distance/velocity relation flips back.
Tingay et al. (1995) presented new radio VLBI and $A T C A$ data of GRO J1655-40. It is the only true work studying the lower distance limit of GRO J1655-40. Firstly, we note that a rather large distance is expected by the authors in order to agree with: "a significant reddening due to absorption", as explained in della Valle (1994). della Valle (1994) is a IAU Circular stating nothing more than: "[...] The [optical] spectrum exhibits prominent, broad Balmer lines [...] superimposed on a relatively red continuum. [...]" However, the spectrum has been taken during a flaring state. The spectral type of the secondary, and its possible veiling by the accretion disk, were, at that time, unknown. Leaving this aside, let us concentrate on the radio spectrum.

The HI spectrum of Tingay and collaborators, obtained with ATCA (see their Fig. 2), shows a multicomponent profile, with strong features at $\sim+5 \mathrm{~km} \mathrm{~s}^{-1}$, between -10 and $-20 \mathrm{~km} \mathrm{~s}^{-1}$, and isolated weak features at -30 and $-50 \mathrm{~km} \mathrm{~s}^{-1}(\sim 18 \%$ and $\sim 2 \%$ of the normalized continuum flux respectively). It is said that the latter feature is confirmed with multiple observations but no references are given.

According to Tingay et al. (1995), the feature at $-50 \mathrm{~km} \mathrm{~s}^{-1}$ would imply a lower limit of $\sim 4.2 \mathrm{kpc}$ if it was participating to the mean galactic rotation ${ }^{2}$. This feature is actually discarded by Tingay and collaborators because it cannot be ruled out that such feature is driven by an expanding shell surrounding the Scorpius OB1 association located at $1.9 \mathrm{kpc}$ from the Sun (see Fig. 1). This is however the feature used by McKay and Kesteven (1994) to derive an approximate distance of $3.5 \mathrm{kpc}$. In other words, McKay and Kesteven (1994) derive $D \sim 3.5 \mathrm{kpc}$ thanks to the feature at $-50 \mathrm{~km} \mathrm{~s}^{-1}$ that is discarded by Tingay et al. (1995) because it would imply $D \gtrsim 4.2 \mathrm{kpc}$.

According to Tingay et al. (1995), the feature at $-30 \mathrm{~km} \mathrm{~s}^{-1}$ implies a lower limit of $3.0 \mathrm{kpc}$ if it is associated with the mean galactic rotation. To strengthen their conclusion, they compare their spectrum with that of a nearby region GRS $345.4+1.4$ (a.k.a. CTB 35 A) studied by Caswell et al. (1975) and located $2.4 \mathrm{kpc}$ away. In fact, the lower limit of the distance of GRO J1655-40 is built on this hypothesis: the feature at $-30 \mathrm{~km} \mathrm{~s}^{-1}$ in the radio spectrum is moving with the mean galactic rotation scheme, which is confirmed by the comparison with GRS 345.4+1.4.

But if there is such uncertainty on the interpretation of the feature at $-50 \mathrm{~km} \mathrm{~s}^{-1}$, why can't the

\footnotetext{
${ }^{2}$ We assumed that the authors follow the same galactic model of Caswell et al. (1975) which is not explicitly stated but whose results are explicitly used.
} 


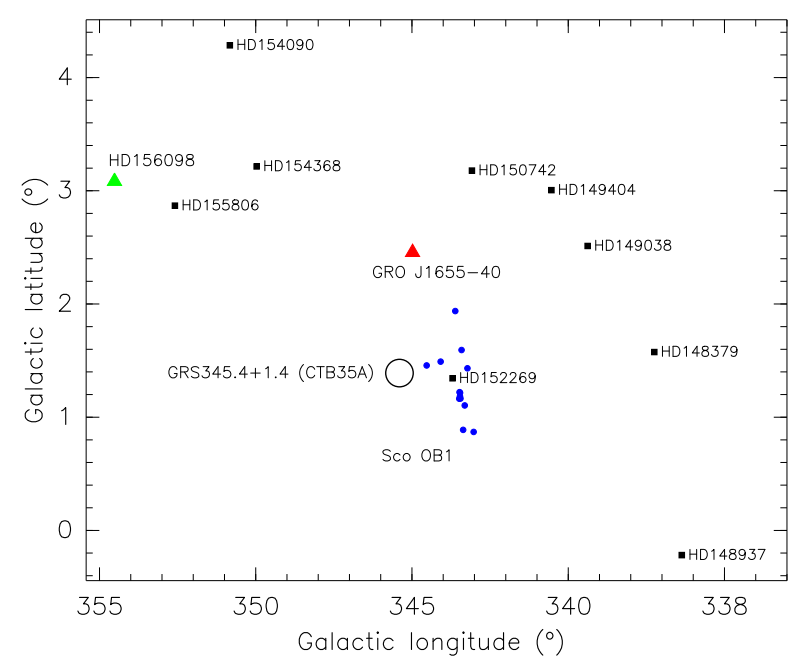

Figure 1: Map of the region around GRO J1655-40, where the region GRS345+1.4 studied by Caswell et al. (1975) is also shown as a big open circle. The 24 stars studied by Crawford et al. (1989) are indicated (stars belonging to Sco OB1 in blue circles, field stars in black squares with names). It shows that GRO J1655-40 is located at the core of the region studied by Crawford and collaborators. Note the presence of the star HD 152269 which lies close to the center of Sco OB1 but is a foreground star. The green triangle to the left shows the position of the comparison F6IV star HD 156098 used by Foellmi et al. (2006) in their analysis.

feature at $-30 \mathrm{~km} \mathrm{~s}^{-1}$ not also be associated with Sco OB1? Or could the latter be indeed associated with Sco OB1 while the most negative one is not? The spectrum of GRS $345.4+1.4$ looks indeed similar to that of GRO J1655-40, except that it has no absorption feature with velocities more negative than $-24 \mathrm{~km} \mathrm{~s}^{-1}$. But as shown on the map in Fig. 1. GRS345.4+1.4 is closer to Sco OB1 than to GRO J1655-40. Why does the spectrum of GRS345.4+1.4 shows nothing more negative than $-24 \mathrm{~km} \mathrm{~s}^{-1}$ although it is angularly closer but still behind the association compared to the position of GRO J1655-40?

We note moreover that Caswell et al. (1975) confirm the results of Radhakrishnan et al. (1972) that the HI absorption spectrum of the region GRS 345.4+1.4 is problematic since either the foreground absorbing cloud at $V=-24 \mathrm{~km} \mathrm{~s}^{-1}$ or the HII region behind it (which is responsible for the continuum emission) has a "peculiar" motion of $\sim 10 \mathrm{~km} \mathrm{~s}^{-1}$. Caswell et al. (1975) determine a (near kinematic) distance of $2.4 \mathrm{kpc}$ by assuming a mean velocity of the multi-profile $\mathrm{HI}$ absorption of $\sim-20 \mathrm{~km} \mathrm{~s}^{-1}$. An uncertainty of $10 \mathrm{~km} \mathrm{~s}^{-1}$ translates to an uncertainty of about $1.5 \mathrm{kpc}$ in distance, if one uses the scale in Caswell et al. (1975 ${ }^{3}$ Furthermore, Shaver

${ }^{3}$ We note that Caswell and coworkers use actually the galactic et al. (1982) have shown that there are a number of HI clouds in this region that have a peculiar velocity which cannot be accounted for by assuming that such cloud is moving with the mean galactic rotation, since the derived distance from the HI absorption appears larger than that derived from optical observations of HII regions along the same line of sight. We are therefore entitled to conclude that no meaningful comparison can be made between two absorption radio spectra in this region of the sky and the interpretation of the negative velocity features in the absorption radio spectrum is uncertain.

We conclude that the lower limit of $3.0 \mathrm{kpc}$ on the distance of GRO J1655-40 has not been established.

\subsection{Optical spectroscopy of stars in the direction of GRO J1655-40.}

Crawford et al. (1989) have studied the interstellar sodium and calcium absorption lines towards the Scorpius OB1 association with high-resolution optical spectroscopy. The stars observed in this study are also shown in Fig. 1. They observed that all the spectra of Sco OB1 members show structured features in the sodium doublet lines with velocities spanning a range as wide as 40 to $60 \mathrm{~km} \mathrm{~s}^{-1}$, to the contrary of all other field stars, including the foreground star HD 152269 located right at the center of Sco OB1 on the sky. The absorption features at the bluer wavelengths inside the absorption lines are interpreted as truly blueshifted because the spectra are calibrated with atmospheric water lines directly on the spectra (i.e. the zero-point in velocity is known).

Crawford et al. (1989) made the global following conclusions, despite variations between individual sources. Firstly, absorption lines comprised in the range $-20 \leq v_{\text {helio }} \leq 0 \mathrm{~km} \mathrm{~s}^{-1}$ must arise from material between Sco OB1 and the observer, since it cannot participate to the mean rotation curve. Second, the absence of blueshifted absorption features with $v_{\text {hel }} \leq-20 \mathrm{~km} \mathrm{~s}^{-1}$ in the spectrum of HD 152269 implies that the absorbing material responsible for these blue features is comprised between the star (720 pc away), and the cluster. Given an average velocity of Sco OB1 of about

model of Schmidt (1965) with a galactic center distance of $10 \mathrm{kpc}$, while the modern value is about $8.0 \mathrm{kpc}$ (Groenewegen et al. 2008). The extraction of the velocity-distance relationship directly on the figure of Caswell et al's paper reveals that the printed scale does not correspond exactly to the results written in the text itself (a velocity of $-24 \mathrm{~km} \mathrm{~s}^{-1}$ correponds to $2.8 \mathrm{kpc}$ instead of $2.4 \mathrm{kpc}$; possibly causing the confusion). However, the use of a smaller galactic center distance roughly compensate this error on the velocity-distance relationship. 

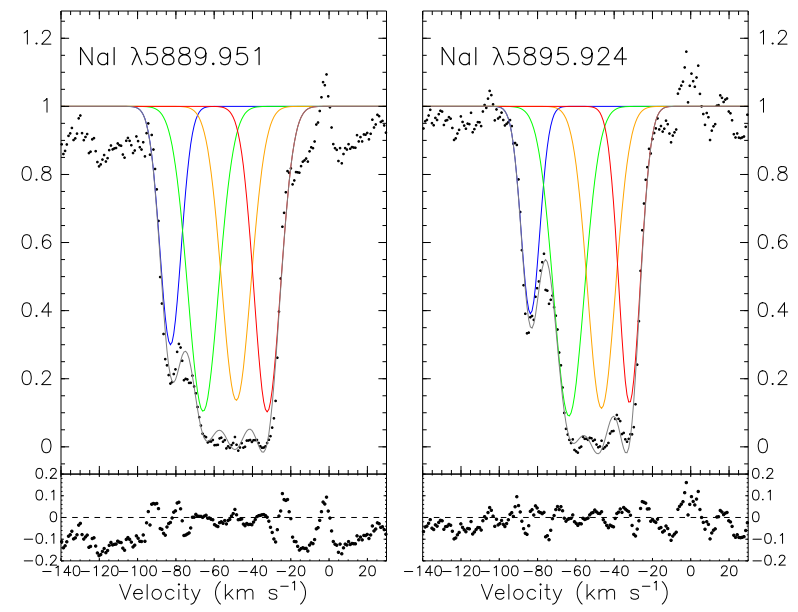

Figure 2: The sodium doublet observed in the spectrum of GRO J1655-40 as function of the heliocentric velocity. Rest wavelength of the sodium were used: $\lambda \lambda 5889.951,5895.924$. The spectra were taken from Foellmi et al. 2006, and were combined together without preliminary radial-velocity shift. The zero-point of the velocity scale in the figure is simply that of the rest wavelength of the sodium lines, to which the calibration of the spectrum agree within $5 \mathrm{~km} \mathrm{~s}^{-1}$ (see text). Four gaussians were fitted to each independent line. The top panels show the spectra (points), with the individual gaussians in color, and the total gaussian in gray line. The bottom panels shows the difference between the spectrum and the fit.

$-25 \mathrm{~km} \mathrm{~s}^{-1}$, the authors conclude that the absorptions seen at the bluest wavelengths of the sodium doublets are caused by expanding material associated with the cluster.

In order to compare with GRO J1655-40, we have taken the original spectra published in Foellmi et al. (2006), and averaged them with no radial-velocity shifts prior to the combination, in order to build a mean spectrum where the (static) interstellar lines are well averaged. The spectrum is shown in Fig. 2. Our UVES spectra are well aligned in a relative manner, since all sharp reddest wings of the $\mathrm{Na}$ lines appeared well aligned to each other. Moreover, we have identified about 10 (residual) sky lines inside the averaged spectrum (also identified in Foellmi et al. 2006). When compared to the skylines atlas of Osterbrock et al. (1996), the velocity zero-point our the spectrum appears to be correct within $5 \mathrm{~km} \mathrm{~s}^{-1}$. We have fitted the interstellar lines with four gaussians. We note the differences, in velocity, between the bluest and the reddest gaussians are 51 and $52 \mathrm{~km} \mathrm{~s}^{-1}$ respectively for the two lines.

We can see in Fig. 2 that there is no absorption between 0 and $\sim-30 \mathrm{~km} \mathrm{~s}^{-1}$. Along with the conclusions of Crawford et al. (1989), it would mean that there is a very small amount of absorbing material between GRO J1655-40 and the observer. This conclusion is hardly reconcilable with a large distance (and afortiori larger than that of Sco OB1). Moreover, the sodium absorption is saturated between -20 and $-60 \mathrm{~km} \mathrm{~s}^{-1}$ and a weaker blue feature exist at $\sim-85 \mathrm{~km} \mathrm{~s}^{-1}$. It seems more likely that these absorptions arise from an expanding material related to GRO J1655-40 itself. As matter of fact, Combi et al. (2007) have investigated the region of GRO J1655-40 with radio and infrared data tracing the $\mathrm{HI}, \mathrm{CO}$, and the gas morphology of the region. They have found that there is evidences of an HI hole of $1^{\circ} .5 \times 1^{\circ} .5$ in diameter at a distance of $D=1.2 \pm 0.4 \mathrm{kpc}$, and compressed CO material accumulated in part of the shell border, as well as infra-red emission with characteristics of shocked-heated dust. This study strengthen the suggestion that GRO J165540 could be located around $1.0 \mathrm{kpc}$, and possibly originated from NGC 6242.

\section{The difficulties in estimating the extinction to- wards GRO J1655-40}

After the value of $3.2 \mathrm{kpc}$ had been published, a significant number of studies confirmed or strengthened this result, by using various distance methods. We discuss in the following sections the various problems encountered when estimating the extinction towards GRO J1655-40.

\subsection{The validity range of the sodium equivalent width- color excess relationship}

A relationship exists between the equivalent width of the sodium doublet or the calcium lines in an optical spectrum, and its color excess. This relationship has been used, for instance, by Bianchini et al. (1997) for GRO J1655-40, although using spectroscopy of low resolution preventing them to see the saturated and multi-profile nature of the lines (see Foellmi et al., 2006, for the details, and Fig. 2 above). They use the relationships given by Herbig (1975) for the calcium line at $6613 \AA$ (see Fig. 4 of Herbig 1975) and della Valle and Duerbeck (1993) (who actually use the photoelectric photometry of Cohen, 1975) for the sodium doublet at $5980 \AA$ :

$$
E(B-V) \sim 0.61 \times E W_{\mathrm{NaI}-\mathrm{D}}-0.08
$$

Bianchini et al. (1997) obtained a color excess ranging from 0.97 to $1.30 \mathrm{mag}$, from which they adopt a mean value of 1.13 , and state that it is in agreement with the value found by other studies on this target (for e.g. Bailyn et al., 1995a, but see below). But such a range on the color excess translates, using a standard value of 
$R=3.1$, to an uncertainty of one magnitude on the absorption $A_{V}$ and therefore a factor of 1.6 in the distance.

Moreover, Munari and Zwitter (1997) have shown that the unambiguous range between equivalent widths of $\mathrm{NaI}$ lines and $E(B-V)$ is $0 \leq E(B-V) \leq 0.4$, i.e. much lower than the value measured by Bianchini and coworkers. If one used the (scattered) relation of della Valle and Duerbeck (1993), the limiting range of $\mathrm{Mu}$ nari \& Zwitter translates into equivalent widths between 0.13 and $0.79 \AA$ i.e. with an upper limit much lower than the value of $2.26 \AA$ from Bianchini et al. Moreover, assuming a single gaussian profile, it implies 4 a FWHM of the lines between 0.12 and $0.75 \AA$, or a corresponding resolving power of about 50000 for the narrowest $\mathrm{Na}$ lines, and 8000 for the broadest. It means that Bianchini and coworkers simply do not have the resolution necessary to derive an accurate value of the color excess to confirm the value of $3.2 \mathrm{kpc}$.

\subsection{Six extragalactic supernovae}

A distance "of $\sim 3.0 \mathrm{kpc}$ " was proposed on the basis of optical data by Bailyn et al. (1995a). The authors have also measured the equivalent widths of NaI-D lines in their spectrum $(\mathrm{EW}=4.5 \AA)$ that has a resolution of $\sim 10 \AA$ (which is even worse than that of Bianchini et al. 1997, and moreover blended with HeI emission). They finally compute a color excess $E(B-V)=1.15$, using the relation between the equivalent width and the color excess given by Barbon et al. (1990). The latter paper studies the type-Ia supernova SN1989B in NGC 3627. Barbon et al. (1990) determined an empirical and roughly linear relation between the equivalent width of the NaI-D lines and the color excess $E(B-V)$ with the spectra of (only) six extragalactic supernovae. Their relation reads (see the end of their section §3.2):

$$
E(B-V) \sim 0.25 \times E W_{\mathrm{NaI}-\mathrm{D}}
$$

defined for $E(B-V)$ between 0.1 and 1.0, after removing the galactic contribution. Leaving aside the intrinsic difficulty of determining the galactic contribution, the source of the data used to determine this relationship is not given in Barbon et al. (1990), and we can question the reliability of a relation calibrated with 6 points only. Moreover, using the relation given by della Valle and Duerbeck (1993), equation 11 would give $E(B-V)=$ 2.66 , instead of 1.15. It implies an absorption value of $A_{V}^{\mathrm{opt}} \sim 8.24$ instead of 3.56 , and therefore a factor of 8.7 in the relative value of the distance (equation 2). Even

\footnotetext{
${ }^{4} \mathrm{FWHM}_{\mathrm{Gauss}}=2 \sqrt{2 \ln 2} \cdot \sigma$ where $\sigma$ is the gaussian width.
}

ignoring the line saturation in the case of GRO J165540 , the two relationships are still hardly reconcilable.

Interestingly, Bailyn et al. (1995a) claimed that their result "is consistent with the EW of other interstellar lines in the optical domain". Finally, they use the classical relationships of Allen (1973, discussed below) and Herbig (1975, who actually did not study the sodium doublet), to conclude that the distance of the source is compatible with $D \sim 3 \mathrm{kpc}$, "in agreement with the radio observations" of Tingay et al. (1995).

\subsection{Where is the HST/STIS spectrum?}

Orosz and Bailyn (1997) presented an extended spectroscopic and photometric dataset. In particular, they have found very clear ellipsoidal variations in their BVRI lightcurves, obtained in February and March 1996 , when the system was not yet completely in quiescence. Although they mention various consistency checks throughout the paper, they do not measure the distance, but rather rely on that of Hjellming and $\mathrm{Ru}-$ pen (1995), said, along with $E(B-V)$, to be "tightly constrained". As for the color excess, they assumed a value of $E(B-V)=1.3 \pm 0.1$, actually obtained by Horne et al. (1996) who used high-quality UV spectra obtained with the Hubble Space Telescope. However, Horne et al. (1996) is an IAU circular where the spectrum is not visible, and where it is simply stated that "deep 220-nm absorption in the HST spectrum suggests $E(B-V)=1.3$ mag."

Similarly, van der Hooft et al. (1998) presented new $V R i$ photometric data acquired during 28 consecutive nights in March 1996 with the Dutch 0.91m telescope in La Silla (Chile), when the source was said to be close to its quiescence brightness (but see below). From the modelling of the lightcurve they obtain a inclination angle of about $67^{\circ}$ and a black hole mass between 6.3 and 7.6 $M_{\odot}$ (consistent with Orosz and Bailyn, 1997). However, they have assumed again the radio distance of $3.2 \mathrm{kpc}$ by Hjellming and Rupen (1995), and a color excess of $E(B-V)=1.3 \mathrm{mag}$, taken from Horne et al. (1996). Interestingly, they mention that the distance is well constrained since it has been found consistent with the results of McKay and Kesteven (1994), Tingay et al. (1995), Bailyn et al. (1995a) and Greiner et al. (1995). We have shown above how weak were these first three references concerning the distance of GRO J1655-40 (the fourth and last reference is discussed in Sec. 4).

\subsection{A new and lower value of the color excess of GRO J1655-40}

One can directly estimate the color excess of GRO J1655-40. As mentioned in Foellmi et al. (2006), 
the F6IV comparison star HD 156098 has a known Hipparcos distance of $50 \pm 0.2$ parsec. It is close enough to assume that it has a negligible interstellar absorption (see for instance Welsh et al., 1990). Therefore we assume that its observed color index is equal to its intrinsic color index: $(B-V)_{F *} \equiv(B-V)_{F *, 0}=0.46$ [using SIMBAD; see also Fitzgerald (1970) who give $(B-V)=$ 0.46 for an F6IV]. Using the mean visual magnitude $V=17.12$ from Orosz and Bailyn (1997) and adopting $B \sim 18.6^{5}$ from their lightcurve, the color index of GRO J1655-40 reads: $(B-V)_{\text {GRO }}=1.48 \mathrm{mag}$. Since Foellmi et al. (2006) have shown that HD 156098 represents a fairly good twin to the spectrum of GRO J1655$44^{6}$ the color excess of the microquasar simply follows: $E(B-V)=(B-V)_{\mathrm{GRO}}-(B-V)_{F *, 0}=1.02 \mathrm{mag}$, which is smaller than previous values. This conclusion is also reached by Beer and Podsiadlowski (2002) who mention that the value $E(B-V)=1.3$ is not consistent with a F6IV star but rather an A8 or earlier. The value of $(B-V)_{F *, 0}$ is a bit outside the validity range of equation 5. If we nevertheless use this equation, we derive $R=3.42$ and therefore $A_{V}=3.49$. The implications of these new values to the luminosity and mass ratio of the GRO J1655-40 are discussed in Sec.5.

\section{The optical absorption derived from X-ray or op- tical data}

We have seen above some difficulties at determining the optical absorption towards a source. This optical absorption can in practice also be obtained from $X$-ray data. We present here the issues related to that approach and its application to GRO J1655-40. In particular, we show that no systematic overestimation from X-ray data when compared to optical data can be claimed.

\subsection{Is the optical absorption of GRO J1655-40 from ROSAT flawed?}

Greiner et al. (1995) presented new ROSAT X-ray data of GRO J1655-40, from which they infer a distance of $3 \mathrm{kpc}$ (no uncertainties are provided). Their method consists of fitting the halo of the observed radial profile of the source. This halo is produced by the scattering of the X-rays by the interstellar dust. To fit the radial profile of GRO J1655-40 observed with ROSAT,

\footnotetext{
${ }^{5}$ There is an error in Foellmi et al. 2006) who use $B \sim 16.65$ instead of $B \sim 18.6$.

${ }^{6}$ We emphasize here that the spectra look the same, i.e. the global average spectroscopic parameters are comparable. We obviously do not mean that the stars are identical. This distinction is important in the next sections.
}

they assume an uniform dust distribution between the observer and the source in their model. They obtain, with not many details, a value of the effective optical depth at $1 \mathrm{keV}$ of $\tau_{\text {eff }} \sim 0.33$.

Furthermore, they use the results of Predehl and Schmitt (1995) who have studied in details X-ray halos in ROSAT sources, and have shown that a good correlation exists between the simultaneous measured dust and hydrogen column densities: "indicating that gas and dust must be to a large extent cospatial". From the fractional halo intensity it is thus possible to derive the dust column density. The relation used by Predehl and Schmitt (1995) reads (see the end of their Sec. 3.4):

$$
\tau_{\text {eff }} / A_{V}=0.056 \pm 0.01
$$

where $A_{V}$ is the visual absorption (expressed in magnitude).

Using this correlation, and assuming that the sightline for GRO J1655-40 has the same gas-to-dust ratio as the sight-lines for which the relations between $A_{V}$ and $N_{H}$ have been established, Greiner et al. (1995) obtain for GRO J1655-40 an absorption of $A_{V}=5.6 \mathrm{mag}$ (and a hydrogen column density of $N_{H}=7.0 \times 10^{21} \mathrm{~cm}^{-2}$, implying a color excess of $E(B-V) \sim 1.7$, assuming $R=3.1$ ). This is significantly (by $\sim 1.5-2$ magnitudes) larger than any other estimation from the studies in the optical $\left(A_{V} \sim 3.8-4.1 \mathrm{mag}\right)$ and our own estimate above.

To finally compute the distance, Greiner et al. (1995) use the mean extinction law given by Allen (1973. Sec. 125, p.263): $A_{V}=1.9 \mathrm{mag} \mathrm{kpc}^{-1}$, and say that it is in agreement with other determinations of the distance. It is however hard to consider the relation of Allen more than a basic approximation, since it is composed of two parts: $A_{V}=1.6 \mathrm{mag} \mathrm{kpc}^{-1}$ from "interstellar absorbing clouds" and $A_{V}=0.3 \mathrm{mag} \mathrm{kpc}{ }^{-1}$ from "grains between the clouds". The value of $1.6 \mathrm{mag} \mathrm{kpc}^{-1}$ is actually obtained by simply assuming that there are 5 clouds per $\mathrm{kpc}$ in the galactic plane, and that the mean visual absorption is about $0.3 \mathrm{mag}$ per cloud (Allen, 1973. Sec. 124 , pp.262-263). The value of $0.3 \mathrm{mag} \mathrm{kpc}^{-1}$ is quoted from Gottlieb and Upson (1969) who in fact clearly show, by dividing the sky into more than 200 zones, that the extinction in a particular direction is by far more complicated than such a simple mean relation.

In summary, the value of the optical absorption determined by Greiner et al. (1995) is intriguing and significantly larger than what we obtained above: $A_{V}=3.49$. On the other hand, their "confirmation" of the distance cannot be trusted, even if, mentioning the $3-5 \mathrm{kpc}$ range of Tingay et al. (1995), they claim in their conclusions 
that: "The scattering of X-rays by the interstellar dust allows to derive a distance of GRO J1655-40 of $3 \mathrm{kpc."}$

\subsection{Is there a systematic effect?}

The significantly larger value of $A_{V}^{\mathrm{X}-\text { rays }}$ compared to $A_{V}^{\text {optical }}$ is important since it can provide strong insights into the role of dust, and the possible superabundance of dust over hydrogen close to the source itself. It is not only an important point for the distance determination, but also for our understanding of the closeby environments of such objects and therefore deserves to be verified.

Jonker and Nelemans (2004) claim that optical absorption obtained from X-rays are systematically larger than those obtained from optical data, as shown in their Table 3 , in which the values of $A_{V}^{\text {optical }}$ and $A_{V}^{\mathrm{X} \text {-rays }}$ are listed for 14 sources, among which GRO J1655-40 and A0620-00. Taken at face value, four targets in the list of 14 sources don't have a value in one of the two bands (an "X" is marked in its place): GS 100945, XTE J1118+480, H 1705-250, SAX J1819.3-2525. Moreover, five others have, strictly speaking, consistent values either because the values agree within the (large) uncertainties or because only upper/lower limits were determined (GRO J0422+32, A0620-00, GRO J165540, GX 339-4 and GS 2 Section 023+338), leaving 5 systems only for a true comparison: GS 1124-684, 4U 1543-47, XTE J1550-564, XTE J1859+226 and GS $2000+25$.

\subsubsection{GS 1124-684}

For GS 1124-684 (=GU Mus, Nova Mus 1991), Cheng et al. (1992) determine the color excess to be $E(B-V) \sim 0.29$, which corresponds to $A_{V}^{\text {optical }}=0.9 \pm$ 0.1 , by fitting a model of a $H S T / F O S$ spectrum between 1600 and $4900 \AA$.

The value of $A_{V}^{\mathrm{X} \text {-rays }}=1.28 \pm 0.06$ is computed by Jonker and Nelemans (2004) taking $N_{H}=2.28 \times 10^{21}$ $\mathrm{cm}^{-2}$ of Greiner et al. (1994a) and using the relation $N_{H} / A_{V}=1.79 \times 10^{21} \mathrm{~cm}^{-2}$ by Predehl and Schmitt (1995 7 , corresponding to a color excess of $E(B-V) \sim$ 0.4. But Greiner et al. (1994a) write that their estimation of the color excess is confirmed by the works of, first, della Valle et al. (1991), and second, Cheng et al. (1992) which is the reference for $A_{V}^{\text {optical }}$. Moreover, della Valle et al. (1991) use the problematic 6-extragalactic supernovae relation of Barbon et al. (1990) discussed above and adopt a mean value for the color excess derived

\footnotetext{
${ }^{7}$ It is actually misspelled "Schmidt" in the caption of Table 3 of Jonker \& Nelemans.
}

from a range of values between 0.2 and 0.35 , i.e. in agreement with that of Cheng et al. (1992).

As mentioned by Greiner et al. (1994a) "the reddening of the X-ray transient in Muscae was first derived to $E(B-V) \approx 0.2-0.3$ from IUE and optical measurements", and give three references, among which two IAU Circulars by West et al. (1991, but see also Appendix A, and Gonzalez-Riestra et al. (1991). Unfortunately, none of the two circulars report a value of the color excess as such. The third reference given is a conference proceeding of Shrader C. and Gonzalez-Riestra R. 1991 (in "Workshop on Nova Muscae 1991", Lyngby 1991, DRI Prep. 2-91, p.85) which is unfortunately not referenced in the NASA ADS system.

We can safely discard the source GS 1124-684 in Table 3 of Jonker and Nelemans (2004).

\subsection{2. $4 U 1543-47$}

For 4U 1543-47 (=IL Lup*), the optical value $A_{V}=$ $1.55 \pm 0.15$ is quoted from Orosz et al. (1998) who also mention that their value is smaller than that derived from X-rays, quoting the values of Greiner et al. (1994b) and van der Woerd et al. (1989) which is the reference for $A_{V}^{\text {X-rays }}$ used by Jonker and Nelemans (2004).

Strictly speaking, Orosz et al. (1998, see their Sec. 4) have the following range $0.45<E(B-V)<0.55$, while the values of the color excess obtained from $\mathrm{X}$ ray data ranges from " 0.56 to 0.77 assuming $A_{V}=$ $N_{H} / 1.79 \times 10^{21}$ (Predehl and Schmitt, 1995)." That is, the two ranges almost agree. Jonker and Nelemans (2004) ignore the value of Greiner et al. (1994b) and choose the upper limit to derive $A_{V}^{\text {optical }}=2.4 \pm 0.1$. Given the difficulties of obtaining reliable estimates in both energy regimes, this disagreement between $A_{V}^{\text {optical }}$ and $A_{V}^{\mathrm{X}-\mathrm{rays}}$ is not really conclusive.

\subsubsection{GS $2000+25$}

For GS 2000+25 (=QZ Vul, Nova Vul 1988), the value from $\mathrm{X}$-ray observations quoted by Jonker and Nelemans (2004) is $A_{V}=6.4 \pm 1.0$, citing Tsunemi et al. (1989). However, in this latter paper, the value quoted is $A_{V}=4.41$ (or $\log _{10} N_{H}=22.06 \pm 0.006$, see their Sec. III, a, iii), said to be in agreement with the optical estimation by Chevalier and Ilovaisky $\left(1990, A_{V}^{\text {optical }}=3.5\right)$ which is the reference given by Jonker \& Nelemans for the optical value, who also note the large uncertainty of the latter. This source must therefore also be discarded from Table 3.

\subsubsection{XTE J1550-564 and XTE J1859+226}

As for the two remaining sources, namely XTE J1550-564 (=V381 Nor) and XTE J1859+226 
(=V406 Vul), there seems to be a difference between $A_{V}^{\mathrm{X} \text {-rays }}$ and $A_{V}^{\text {optical }}$, but not necessarily because of a systematic effect in one of the two wavelength regime.

For the X-ray binary XTE J1550-564, Jonker and Nelemans (2004) quote $A_{V}^{\text {optical }}=2.5$ (no uncertainties) computed from Sánchez-Fernández et al. (1999) using their value of the equivalent width of the Diffuse Interstellar Band $(\mathrm{EW}=1.9 \AA$, for the $4430 \AA \mathrm{DIB})$ and the relation of Herbig (1975). Sánchez-Fernández et al. (1999) actually report $A_{V}^{\text {optical }}=2.2$ from $\langle E(B-V)\rangle=$ $0.7 \pm 0.2$ using a combination of the $\mathrm{NaI}$ lines and the DIB and using again the 6-supernovae relationship of Barbon et al. (1990) discussed above. Given the uncertainties on $E(B-V)$, the two values actually agree. But if one uses the relationship of della Valle and Duerbeck (1993), $E(B-V)=1.6$, and $A_{V}^{\text {optical }} \gtrsim 5$. In any case, their equivalent width of the $\mathrm{NaI}$ lines of $2.4 \AA$ is much larger than the significant range computed above from the study of Munari and Zwitter (1997): $E W_{\mathrm{NaI}} \approx 0.1-0.8 \AA$ (or $0 . \leq E(B-V) \leq 0.4)$. Moreover, they use a lowresolution ( $2 \AA$ ) spectrum, and it is not clear if the lines are saturated or not, which is the reason why Jonker and Nelemans (2004) use their value of the DIB only.

As for XTE J1859+226, Jonker and Nelemans (2004) mention that $A_{V}^{\mathrm{X} \text {-rays }}=4.47$ has no error bars. This value is quoted from the IAU Circular no. 7291 by dal Fiume et al. (1999) who found $\mathrm{N}_{H}$ to be "about $8 \times 10^{21} \mathrm{~cm}^{-2}$ ". $A_{V}^{\text {optical }}=1.80 \pm 0.37$ is given by Hynes et al. (2002) who found $E(B-V)=0.58 \pm 0.12$ from a spectral fit of the UV feature at $2200 \AA$ in their $H S T / S T I S$ spectrum. Assuming $A_{V}^{\mathrm{X} \text {-rays }}$ being correct, the 2.6 mag difference is certainly due to local absorption in the source itself.

\subsection{Is there a discrepancy between $A_{V}^{X-\text { rays }}$ and $A_{V}^{\text {optical }}$ ?}

To summarize, the claimed systematic overestimation of the optical absorption obtained from X-ray observations compared to the value obtained from optical studies is not established, since only two sources (XTE J1550-564 and XTE J1859+226) among the 14 used by Jonker and Nelemans (2004) potentially show any difference. To claim a systematic effect in one given direction also implies the knowledge of where the correct value is.

The overestimation of $A_{V}^{\mathrm{X} \text {-rays }}$ over $A_{V}^{\text {optical }}$ mentioned by Jonker and Nelemans (2004) has apparently already been observed by Vrtilek et al. (1991). But Vrtilek and coworkers obtained all their absorption values from optical data from an older source: Bradt and McClintock (1983), who do not always measure the absorption themselves but cite even older sources. Moreover, the supposed systematic overestimation of Vrtilek et al.
(1991) is pictured in their Fig. 6a where the non-zero slope showing the effect is caused by 2 deviating points only. According to their own caption, one point is a high-mass X-ray binary (4U 1516-56, a.k.a. Cir X-1) that we know today contains local absorption (Johnston et al. 2001) and where near infrared (NIR) spectra in the $\mathrm{K}$ band revealed itself to be too obscured to allow a spectral classification (Clark et al. 2003) (see also Mignani et al., 2002, for HST data on this source). The second deviating point of the figure is a low-mass X-ray binary (4U 1728-337, a.k.a. GX 354+00) in a globular cluster. The $A_{V}^{\text {optical }}$ of this source is determined by Grindlay and Hertz (1981) not with the mentioned optical data but rather with $J H K$ photometry. Moreover, the values of $A_{V}^{\text {optical }}$ and $A_{V}^{\mathrm{X} \text {-rays }}$ for this object agree within $0.2 \mathrm{mag}$.

We note that two sources (namely XTE J1859+226 and GRO J1655-40) seem to have $A_{V}^{\mathrm{X} \text {-rays }}$ significantly larger than $A_{V}^{\text {optical }}$. Given that most of the X-ray observations are done during outbursts, and it is tempting to think that a local absorber makes the estimation of $A_{V}^{\mathrm{X} \text {-rays }}$ flawed. We note however that the work of Predehl and Schmitt (1995), used by many for their relation between $A_{V}$ and $N_{H}$, show precisely that there is a surprisingly good correlation between the simultaneously measured dust and gas density.

It seems clear that this point should deserve more studies, especially with new methods of determining $A_{V}^{\mathrm{X} \text {-rays }}$. For instance, Xiang et al. (2007) use time delays between photons that are being scattered by dust, and being recorded in the X-ray halo. Interestingly, these delays are also function of the distance to the source.

\subsection{The quiescence state might be variable}

In Jonker and Nelemans (2004), the quoted value of the absorption of GRO J1655-40 is $A_{V}^{\mathrm{X} \text {-rays }}=4.8 \pm 2.8$, which is still in agreement both with the peculiar value of Greiner et al. (1995), and the value of $A_{V}^{\text {optical }}$ from Hynes et al. (1998, $\left.A_{V}^{\text {optical }}=3.7 \pm 0.3\right)$ and our own determination of $A_{V}^{\text {optical }}=3.49$ above. Nevertheless, one additional source of uncertainty is whether the object was observed in quiescence. Jonker and Nelemans (2004) refer to Kong et al. (2002) concerning GRO J1655-40. These authors found a value of the X-ray luminosity measured with Chandra lower than that of Greiner et al. (1995) with ROSAT, and even lower than another measurement made between two large outbursts separated by one year, obtained with ASCA by Ueda et al. (1998) and Asai et al. (1998). Kong and coworkers note that their Chandra observation occurred about 4 years after 
the last outburst, and may therefore measure the quiescent X-ray level more accurately (although this is not necessarily true). In addition, Garcia et al. (2001) emphasize that Wagner et al. (1994) observed variations of the quiescent X-ray flux in V404 Cyg by a factor of 10 with ROSAT.

Finally, the variability in the optical and near-infrared has been recently demonstrated by Cantrell et al. (2008) for when the object is considered in X-ray quiescence. They have studied optical and infrared photometry of A0620-00 spanning the years 1999-2007. They clearly demonstrate the existence of three different states (passive, loop, active) in which only the passive one truly represent quiescence. Therefore, great care must be used when modelling optical lightcurves, since it will have a direct influence on the observed quiescent visual magnitude, and the determination of the Roche-lobe effective radius. These issues are discussed in the next section.

\section{The characterisation of the orbit and the sec- ondary star of GRO J1655-40}

We have shown the various difficulties at determining a robust value of the color excess and/or the absorption value. We now turn to the other problematic term in equation 2, the determination of the absolute magnitude of the secondary star, emphasizing that none of the studies mentioned in this section were aware of the requirement to isolate true optical quiescent lightcurves, as mentioned above.

\subsection{Comparison of height different studies of the orbital and secondary star parameters of GRO J1655-40.}

To obtain the absolute magnitude of the secondary star, we need to combine information on the temperature of the star and information on its radius (see equation 6. There are various problems arising with this approach, which are all exemplified in Table 1 where we extracted the quantitative results on the orbital parameters of GRO J1655-40 and its secondary star obtained by van der Hooft et al. (1997), Orosz and Bailyn (1997), van der Hooft et al. (1998), Phillips et al.(1999), Shahbaz et al. (1999), Greene et al. (2001), Beer and Podsiadlowski (2002) and Shahbaz (2003). All these studies use either optical spectra or CCD photometric lightcurves in the optical domain, or both. Some reanalyse data published previously in order to correct a specific assumption in the given analysis.

In Table 1 we have extracted the relevant orbital and secondary star parameters, being very careful to choose the best quantitative results according to the authors. In the lower part of the table, we have, in addition, computed the projected rotational $v \sin i$ (in $\mathrm{km} \mathrm{s}^{-1}$ ) using the relationship of Horne et al. (1986) when both the mass ratio $q \equiv m_{2} / m_{1}$ (where $m_{1}$ is the mass of the black hole; in Table 1 we use the inverse definition: $\left.Q \equiv m_{1} / m_{2}\right)$ and the secondary radial-velocity semiamplitude $K_{2}$ were available 8

$$
v \sin i=K_{2}(1+q) \frac{0.49 q^{2 / 3}}{0.6 q^{2 / 3}+\ln \left(1+q^{1 / 3}\right)}
$$

We emphasize that this relationship is valid when the hypothesis of synchronous rotation is verified. When the inclination angle was available, we computed the rotational velocity $v_{r o t}$. The Roche-lobe effective radius (in $R_{\odot}$ ) is calculated using the formula of Paczyński (1971) (see also Jonker and Nelemans (2004) for a discussion) from the period $P$ and the secondary mass $m_{2}$ :

$$
R_{2} \equiv R_{\text {Roche Lobe }}=0.234 P_{\text {orb }}^{2 / 3} m_{2}^{1 / 3}
$$

with $P$ the orbital period expressed in hours and $m_{2}$ in solar masses. Given the Roche radius and the orbital period, we computed the equatorial velocity of a star with the same radius, which allow a cross-check of the synchronous rotation hypothesis.

When a temperature was available, we also computed the so-called Roche luminosity, using equation 6 , the Roche radius and assuming a solar temperature $T_{\odot}=$ $5800 \mathrm{~K}$. We then calculated the absolute magnitude $M_{V}$ from this Roche luminosity, using the absorption $A_{V}=3.49$ determined above for GRO J1655-40, and the apparent magnitude $m_{V}$ from the considered data. Finally, we also computed the corresponding distance.

\subsection{Notes on individual studies.}

The paper by Bailyn et al. (1995b) is not discussed because it uses observations that are the first on this target in the optical domain, and as such, provides only very few quantitative results. We also ignore the study by Hynes et al. (1998), who present a large dataset, and discuss extensively the problem of the extinction towards GRO J1655-40, but use basically all dynamical parameters from Orosz and Bailyn (1997). Other studies, such as Buxton and Vennes (2001) or González Hernández et al. (2008) provide updates to a small subset of the orbital parameters, but either have large uncertainties or refer heavily to values determined elsewhere.

\footnotetext{
${ }^{8}$ We note that Shahbaz et al. (1999) and Shahbaz 2003) also use this relationship but there is a typo in their formula: the $1 / 3$ exponent is misplaced.
} 


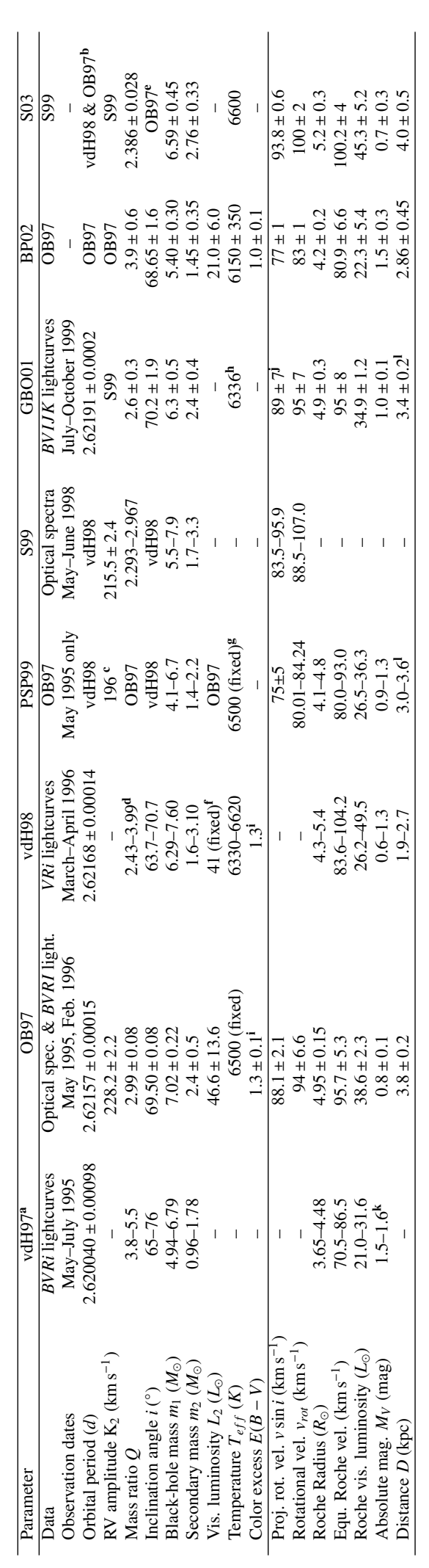

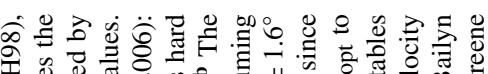

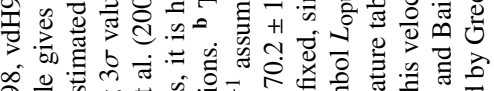

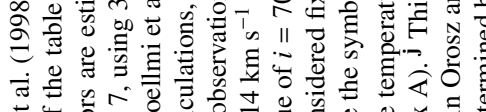

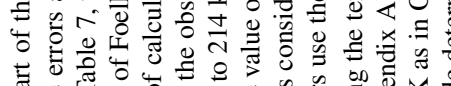

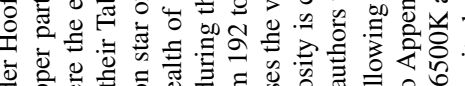

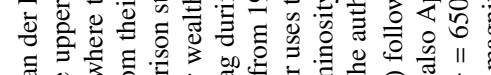

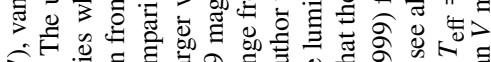
人.

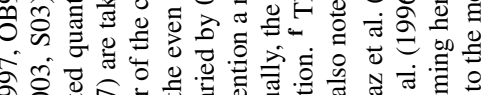

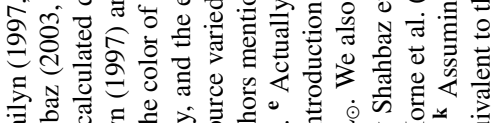

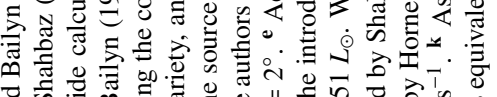

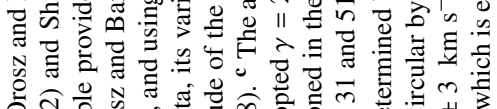

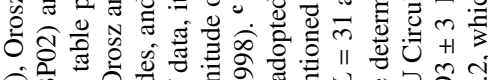

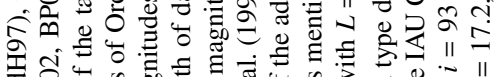

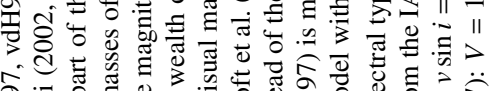

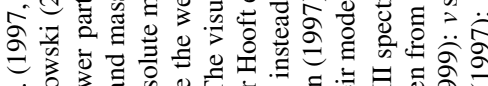

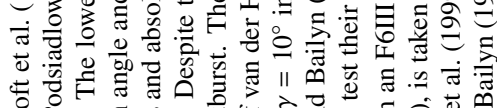

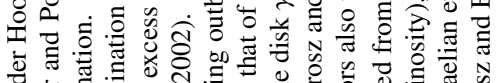

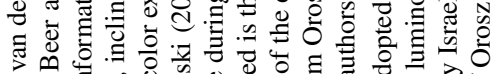
m.

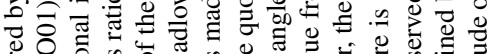

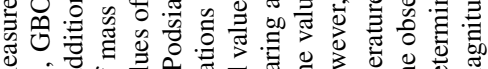

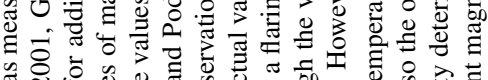

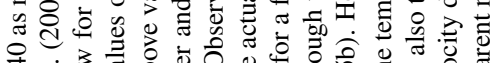

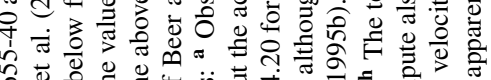

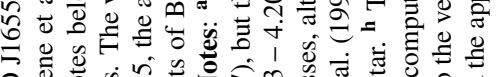

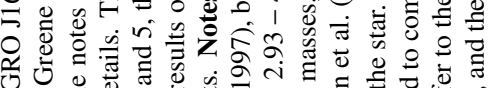

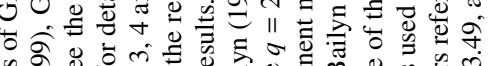

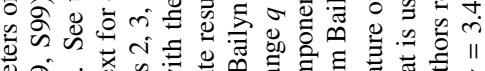

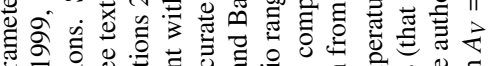

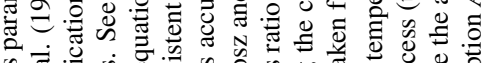

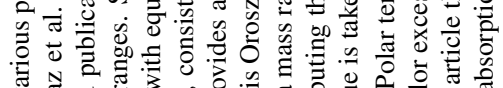

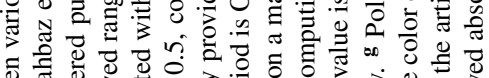

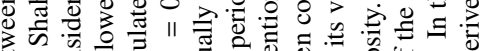

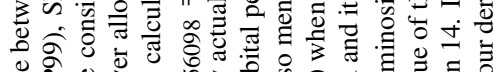
造

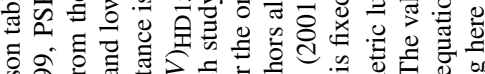

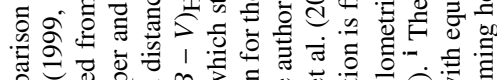

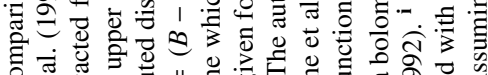

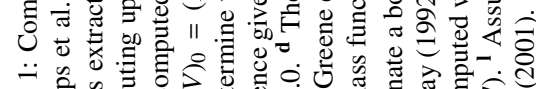

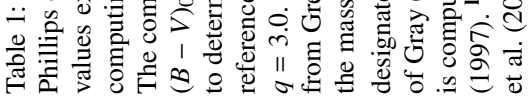


We think that none of the studies mentioned in this section obtained an accurate and complete parameter set about GRO J1655-40. It is nevertheless insightful to excerpt specific issues that compromise the accuracy of the quantitative results.

\subsection{1. van der Hooft et al. (1997)}

The main issue in van der Hooft et al. (1997) is the fact that observations were taken during the outburst, and the overall magnitude increases by 0.9 mag during the course of the observations. Their folded $R$-band lightcurve clearly shows two different levels of maximum (see their Fig. 3). The authors finally determine the mass ratio by fitting their lightcurve with a basic eclipsing-disk model with X-ray heating and two estimations of the X-ray luminosity communicated privately by Harmon. Given the intrinsic variability seen in the data, it is clear that only estimations can be made. With the component masses and the orbital period, it is in principle possible to derive a value for $K_{2}$ and calculate the rotational velocity. However, given the large uncertainties on the mass ratio and the secondary mass, it would be meaningless. We nonetheless note that, if the system is synchronous, the allowed range for the equatorial Roche velocity $\left(70.5-86.2 \mathrm{~km} \mathrm{~s}^{-1}\right)$ is in disagreement with a projected (and hence minimum) rotational velocity of $93 \pm 3 \mathrm{~km} \mathrm{~s}^{-1}$ determined spectroscopically by Israelian et al. (1999) and with which Foellmi et al. (2006) totally agree.

\subsubsection{Orosz and Bailyn (1997)}

Orosz and Bailyn (1997) used optical spectra taken during the outburst (in 1995) and outside the outburst (in 1996), as well as BVRI photometric lightcurves (only in 1996). Their spectroscopic data comprises the data published in Bailyn et al. (1995b). Despite the fact that the source was variable and the secondary star is filling its Roche lobe, they fit a sine wave to their radial velocity data (see their Fig. 1). This issue is corrected later by Phillips et al. (1999, but see below). In order to determine the inclination and the mass ratio, they fit their lightcurves with a model comprising 16 parameters, among which 9 are fixed, and where the spectrum of the star is approximated by a blackbody. This latter issue is corrected in Beer and Podsiadlowski (2002, also discussed below).

Concerning the distance, if we take the observed value of the apparent magnitude $V=17.12$, their luminosity $L=46.6 L_{\odot}$, combined with $E(B-V)=1.3$ and a standard $R=3.1$, we obtain $D=3.1 \mathrm{kpc}$. However, if we take the Roche luminosity (hence assuming synchronicity, as did the authors) inferred from their pre- cise mass $m_{2}$, the orbital period $P$, and the visual absorption value we determined earlier $\left(A_{V}=3.49\right)$, we obtain $D=3.8 \pm 0.2$, i.e. larger than the upper limit determined by Hjellming and Rupen (1995) with the radio jets. Moreover, we note that the value of the luminosity decreases by a factor of two in the corrected model of Beer and Podsiadlowski (2002, see below).

\subsection{3. van der Hooft et al. (1998)}

van der Hooft et al. (1998) attempted to fit their VRi lightcurves with an ellipsoidal model in which the secondary mass $m_{2}$ and the inclination angle $i$ are left free. However, they used a fixed mass function that has been determined with the early data taken by Bailyn et al. (1995b) during outburst. Once the mass function is known, the primary mass, and thus the mass ratio (required for the model) are known. Moreover, they assume a bolometric luminosity (strangely called $L_{\mathrm{opt}}$ in the article) $L=41 L_{\odot}$ taken from Orosz and Bailyn (1997), which does not corresponds to the correct value: $L=46.6 L_{\odot}$. They also assume $E(B-V)=1.3 \mathrm{mag}$ taken from the IAU Circular by Horne et al. (1996) discussed in Sec. 3.3. With such fixed values determined elsewhere from outburst data, it is unlikely that the outcome will be accurate.

In Table 3, we note that the distance implied by their study on the distance cover the range $3.0-4.2 \mathrm{kpc}$, due to the fact that they have a very uncertain $m_{2}$, hence mass ratio, combined with a poorly constrained inclination angle.

\subsubsection{Phillips et al. (1999)}

Phillips et al. (1999) reanalyse the outburst spectroscopic data only of Orosz and Bailyn (1997), ignoring the photometric datasets. They show that X-ray heating of the secondary surface can significantly modify the radial velocity curve. They finally obtain a semiamplitude of $K_{2}=196 \mathrm{~km} \mathrm{~s}^{-1}$, i.e. much lower than the previous value, and therefore derive an "updated" value of the mass ratio. Using the the allowed range for inclination angle determined by van der Hooft et al. (1998), they can compute a new mass for the black hole. This study certainly demonstrates the need to correctly take into account the X-ray irradiation when modelling the radial-velocity curves (or alternatively, re-emphasize the need to choose a truly quiescent optical lightcurve; see again Cantrell et al., 2008). But it does not provide accurate results for the GRO J1655-40 system itself.

We note in Table 1 that their results imply a smaller rotational velocity $v \sin i=75 \pm 5 \mathrm{~km} \mathrm{~s}^{-1}$, which is 
again in large disagreement with the measured value mentioned above.

\subsubsection{Greene et al. (2001)}

Greene et al. (2001) obtained photometric lightcurves in the optical $(B, V$ and $I$, said to be "quantitatively indistinguishable from that of Orosz and Bailyn (1997) and van der Hooft et al. (1998)"). They also obtained for the first time near infrared data (in $J$ and $K$ bands) in order to have better constraints on the role of the accretion disk. Moreover, their modelling is based on the code presented in Orosz and Hauschildt (2000) which is more sophisticated than what Orosz \& Bailyn used. The authors mention that they make use of "all of the binary system observables and their uncertainties", mentioning the 29 points of the radial velocity curve of Shahbaz et al. (1999) as their second dataset (the first one being their own photometry, and the third being the rotational velocity of Israelian et al. (1999)). Table 1 illustrates clearly that the results of Greene et al. (2001) are in perfect agreement with that of Shahbaz et al. (1999), but not with that of Beer and Podsiadlowski (2002) who use basically identical photometric data (see below).

The reason of the discrepancy is provided by Beer and Podsiadlowski (2002) who discuss extensively the results of Greene et al. (2001). Similarly to the work of Orosz and Bailyn (1997), Greene and coworkers implicitly allowed for arbitrary offsets between the different lightcurves, which are not fitted simultaneously (in addition to not using any extinction and distance information). Greene et al. concluded that the accretion disk does not contribute to the lightcurves. On the other hand, Beer and Podsiadlowski (2002) found a much poorer fit of the multi-color data when no disk is present, concluding that no fully self-consistent models can be obtained without a disk.

\subsubsection{Shahbaz et al. (1999) and Shahbaz, (2003)}

Shahbaz et al. (1999) analyse new optical spectra taken during X-ray quiescence and find $K_{2}=$ $215 \mathrm{~km} \mathrm{~s}^{-1}$. The data was then reanalyzed by Shahbaz (2003) who developed a procedure to determine the spectroscopic mass ratio in interacting binaries only by modelling the secondary star spectrum only.

There are various issues with this approach, but the main one is with the data. In the introduction, the author emphasizes the numerous problems associated with the developed procedure (intermediateresolution spectroscopy only, shape of the rotational profile, wavelength-dependent limb-darkening, Rochelobe filling and so on), but the solution proposed to reduce "these uncertainties" is to "determine the exact rotationally broadened spectrum from the secondary star in an interacting binary", i.e. make the best possible model. It remains true however that even a perfect model cannot work well with medium quality data.

As matter of fact, the spectral range is comprised in a very small region between $6320 \AA$ and $6550 \AA$, where there are many iron lines, with a resolution of $4.2 \AA$. With an observed rotational velocity of $93 \mathrm{~km} \mathrm{~s}^{-1}$ (see above), the line are heavily blended. However, an intermediate step of the procedure involves fitting the continuum of the spectrum. This issue is crucial, since the procedure rely on the strength of the lines, which obviously depends critically on the true continuum level and the amount of possible veiling by the accretion disk. There is no detailed indication about this central step, apart that a spline fit is made before combining the spectra (which is also not an easy task). This issue is already present in the analysis of the abundances in GRO J1655-40 secondary star by Israelian et al. (1999) and which has been questioned in Foellmi et al. (2007) who demonstrate the impossibility to measure abundances as precisely as it has been claimed ${ }^{9}$ Even with a Signal-to-Noise ratio of 100 , it is very difficult to know where is the continuum in the spectrum, if any is truly present given the very broad lines. This requires a spectrophotometric calibration of excellent quality (one or two \% maximum), which is the case neither in Shahbaz et al. (1999) nor in Israelian et al. (1999).

Interestingly, Shahbaz (2003) obtain a poor first fit that they attribute partially to the fact that some elements might be overabundant as it has been supposedly shown by Israelian et al. (1999). Given the amount of uncertainties and the quality of the data used, we can safely discard the results of this study. Moreover, the distance implied by the results is the largest of the height studies compared in Table $1(D=4.0 \pm 0.5)$.

\subsubsection{Beer and Podsiadlowski (2002)}

Beer and Podsiadlowski (2002) reanalyze the photometric data of Orosz and Bailyn (1997), and ignore their spectra. They developed a better lightcurve modelling by not assuming a perfect blackbody spectrum for the secondary star, which has strong consequences on the relative fluxes between the $B$ and the $V$ filter bandpasses. Moreover, they fit simultaneously in a selfconsistent manner the lightcurves in the different passbands. One can see in Table 1 that their results imply

\footnotetext{
${ }^{9}$ The issue has not been truly addressed in González Hernández et al. (2008), where it is still not demonstrated how the continuum location is determined.
} 
a decrease of luminosity by a factor $\sim 2$, and a smaller mass of the secondary star by $40 \%$ compared to that of Orosz \& Bailyn.

The problem in the models of Beer and Podsiadlowski (2002) is that the distance is said to be a free parameter, although it is not clear if it has been allowed to go as low as $1.0 \mathrm{kpc}$. In many places the authors claim that the distance of $3.2 \mathrm{kpc}$ of Hjellming and $\mathrm{Ru}-$ pen (1995) has been used "to tighten" their results, since the three main parameters (distance, color excess and temperature) are highly correlated, and can compensate for each other. But if $D$ is basically fixed, what room is left for the other parameters? Interestingly, Beer and Podsiadlowski (2002) found it reasonable to use a distance of $3.2 \mathrm{kpc}$ since the distance by Hjellming and Rupen (1995) is consistent with the other determination of Bailyn et al. (1995a), Greiner et al. (1995), McKay and Kesteven (1994) and Tingay et al. (1995), although they also note that the existence of a significant difference between the jet inclination $\left(\sim 85^{\circ}\right)$ and the disk inclination angles makes the model of the wiggles in radio jet data not necessarily appropriate. We have shown above how problematic are these references.

Let us mention here that a distance of $1.0 \mathrm{kpc}$ implies a velocity $\beta=0.28$ and consequently an inclination angle of the jets of $\sim 71^{\circ}$. It means that at $1.0 \mathrm{kpc}$, GRO J1655-40 would probably not have its disk misaligned with the jets to the contrary of what has been claimed (e.g. Maccarone, 2002).

Finally, we need to emphasize here that the first bestfit steady-state disk model in Beer and Podsiadlowski (2002) is discarded by the authors because it was giving a distance "much lower than $3.2 \mathrm{kpc}$ ". The work by Beer and Podsiadlowski (2002) is certainly the best multi-color modelling of the lightcurves of GRO J165540 to date. Unfortunately, it remains unknown what the solution of their model was with a much smaller distance.

\subsection{Summary}

Despite the wealth of data, its variety and the even larger wealth of calculations made either on the data itself, or on the interpretation of the quantitative results (evolutionary tracks, Monte Carlo simulations, $\chi^{2}$ distance minimization between spectra, etc.), it is hard to determine which study actually provide accurate, and not only precise, results. It is partly due to the fact that each paper studies only a subset of all parameters, taking the missing ones from other publications.

To summarize, we have clearly demonstrated how difficult the modelling of an interacting binary such as GRO J1655-40 is, and that a complete and consistent modelling of the photometric and spectroscopic datasets taken during true optical quiescence is still lacking.

\section{A new estimation of the distance of GRO J1655- 40}

Recently, Guver et al. (2008) have derived the distance of the galactic neutron star 4U 1618-52 using the fact that red clump giant stars can be considered as standard candles (see López-Corredoira et al., 2002, where the method is well explained). Following this method, we retrieved 2MASS JHK magnitudes of stars in a box of $0.45^{\circ} \times 0.45^{\circ}$ around the position of GRO J1655-40. On the 14659 stars, we constructed a Color-Magnitude diagram (CMD) and selected the red clumps giants in a similar way to what is done in Guver et al. (2008), and as shown in Fig. 3. We obtained 6033 stars that we binned in magnitudes with bins of widths 0.5 mags. For each bin, we fitted a gaussian on the histogram of the number of stars as a function of the color $J-K$. Each gaussian fitted peak provides a value of the color $J-K$, which is then identified to the color of red clump giant Stars of the given magnitude bin (Fig. 4). Identically to Guver et al. (2008), we then assume an absolute magnitude of $K=-1.62$ and a unabsorbed color $(J-K)_{0}=0.7$ for these stars. (See also Durant and van Kerkwijk, 2006, for slightly different values and a little variant to this method.)

By using the general equation 2, the following relationship between the absorption magnitude in the $\mathrm{K}$ band and the color indices:

$$
A_{K}=c_{e} \times\left[(J-K)-(J-K)_{0}\right]
$$

with $c_{e}=0.657$ (see Guver et al.), we can derive a relationship between the distance and the absorption derived from the color, in this direction of the sky (see Fig. 57. We have also derived this relation with stars included in areas of $0.15^{\circ} \times 0.15^{\circ}$ and $1.0^{\circ} \times 1.0^{\circ}$ around the position of GRO J1655-40(70527 stars in total and 25108 stars selected for the box of one degree squared). It does not change the result significantly as shown in Fig. 5. We also made tests on the extent of the selected region in the CMD, and by varying the number, sizes and positions of the magnitude bins, but again it proved to have a negligible influence on the final curves.

The problem is estimating the $(J-K)$ and $(J-K)_{0}$ colours in equation 16 for GRO J1655-40. The former can be visually estimated from the $J$ and $K$ lightcurves by Greene et al. (2001): $J-K \sim 0.55$ (Beer and Podsiadlowski 2002, use $J-K=0.6$ ). As for the later, we need to assume that the infrared color of the secondary star in 


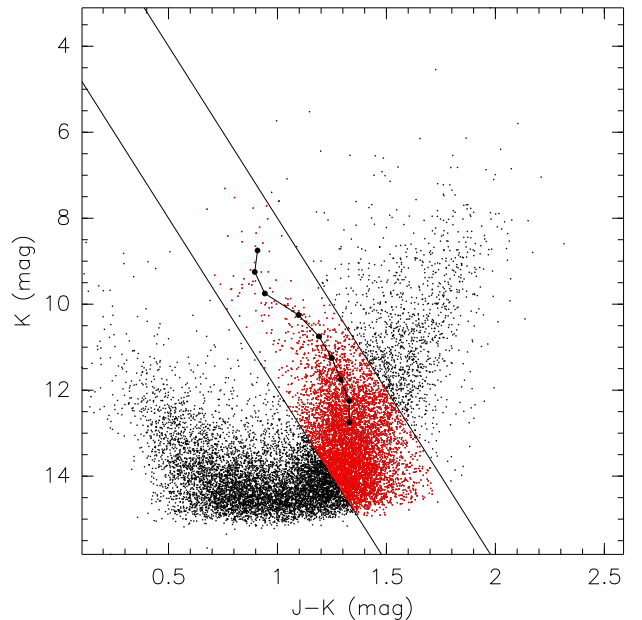

Figure 3: Color-Magnitude diagram of the stars in a square of $0.45^{\circ} \times 0.45^{\circ}$ around the position of GRO J1655-40 extracted from the 2MASS database. The two straight lines delimitate the selected region (red dots). The linked points indicate the maximum number density of selected stars in every magnitude bins. By using a larger area, we increase the number of stars and thus the contamination of the sample. On the other hand, using a smaller area make the results less precise.

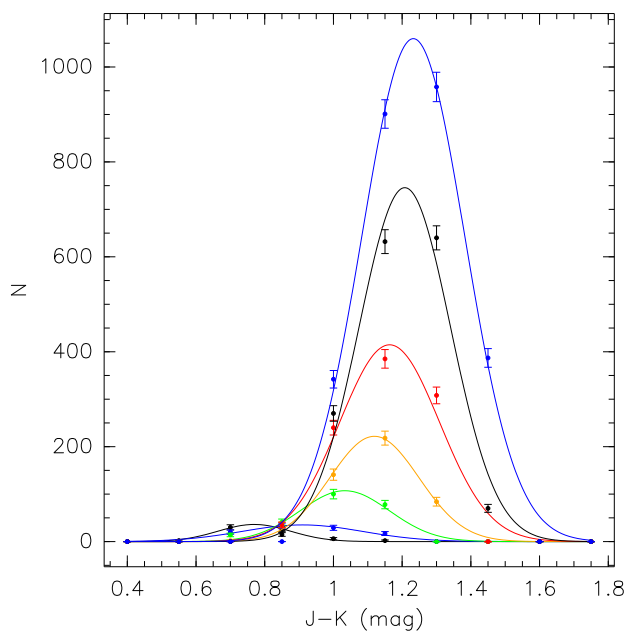

Figure 4: Gaussian fits to the histograms of the number of selected stars for each magnitude bins as a function of the $J-K$ color. One can see that there is a progression of the maximum of the gaussian peaks towards redder colors when we go from bright stars (lower curves with peaks to the left) to fainter stars (upper curves).

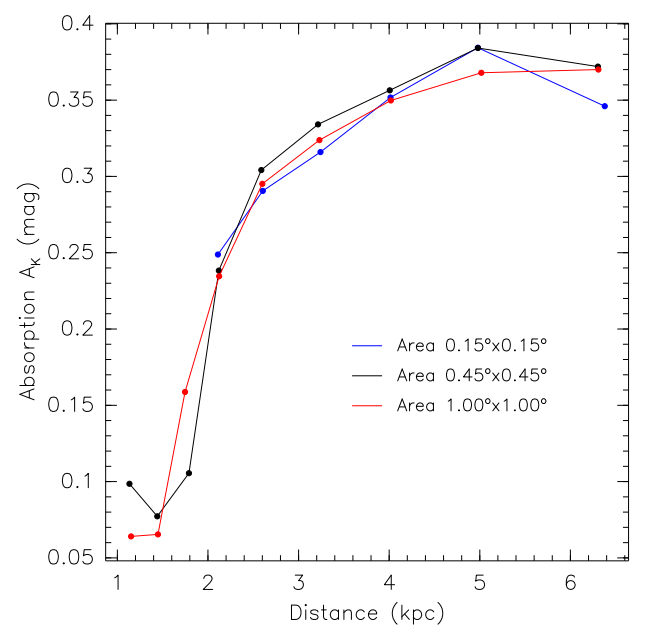

Figure 5: Calculated relationships between the absorption $A_{K}$ and the distance using the selected stars of the three different areas centered around GRO J1655-40. The curves saturate around $D \sim 5 \mathrm{kpc}$, or $A_{K} \sim 0.4$, certainly because of the increased amount of contamination of dwarfs stars and M-giants, as mentioned by Guver et al. (2008) (non-completeness of the 2MASS data for faint magnitudes might also play a role, for the faintest ones). Similarly, at small distance, there are too few stars to obtain a perfectly reliable curve.

GRO J1655-40 can be compared to that of an unaffected star of similar spectral type (F6IV). Koornneef (1983) gave $J-K$ ranging from 0.24 to 0.29 for $\mathrm{F} 2$ to $\mathrm{F} 8 d$ warf stars 10 On the other, one can also use the 2MASS values of the comparison star HD 156098 of Foellmi et al. (2006) which is 50 pc away from the sun and can be considered as unaffected by extinction. Its infrared color is $(J-K) \equiv(J-K)_{0}=0.397$. Using the extreme values of the given range $0.24<(J-K)_{0}<0.4$, and equation 16, we derive a $K$-band absorption $0.1<A_{K}<0.2$ for GRO J1655-40. A comparison with the curves of Fig. 5] shows that the distance of GRO J1655-40 is certainly less than $2.0 \mathrm{kpc}$.

We note however that it is still not possible to definitely conclude the exact distance of GRO J1655-40 (i.e. it lies at the same distance as the cluster NGC 6242) because of the impossibility to derive a reliable curve for the brightest (and thus nearby) red clump giant stars. However, these new results confirm that of Foellmi et al.: GRO J1655-40 is likely to be much closer than currently admitted.

\footnotetext{
${ }^{10}$ No values are given for $\mathrm{F}$ giants, but $J-K$ ranges from 0.22 to 0.35 for $\mathrm{F} 2$ to $\mathrm{F} 8$ supergiants.
} 


\section{The distance of A0620-00}

We have presented above a confirmation that the distance of GRO J1655-40 is certainly smaller than $2 \mathrm{kpc}$. One can thus ask: is GRO J1655-40 the closest (stellar) known black hole to the Sun? According to Jonker and Nelemans (2004), A0620-00 is at a distance of $1.2 \pm 0.4 \mathrm{kpc}$ from the Sun and is so far the closest known black hole to the Sun 11 However, its distance is also problematic, but for different reasons than that of GRO J1655-40. We start by critically review the published distance of A0620-00 before presenting a new estimation of its maximum distance.

\subsection{What is the the color excess towards A0620-00?}

In their study of distances of low-mass X-ray binaries, Jonker and Nelemans (2004) quote Shahbaz et al. (1994) and Barret et al. (1996) for the distance of A0620-00. Shahbaz and collaborators give a distance range between 650 and $1450 \mathrm{pc}$, with a preferred value of $1050 \mathrm{pc}$.

To estimate the extinction toward A0620-00 Shahbaz et al. (1994) use an estimation of the color excess $E(B-V)=0.35$ from $\mathrm{Wu}$ et al. (1983). But $\mathrm{Wu}$ and collaborators actually quote their own results obtained a few years earlier: Wu et al. (1976). This latter paper describe that the extinction is measured by filling the "extinction spectral feature" at $2200 \AA$ in their UV spectrum. However, the spectrum of Wu et al. (1976) has been obtained with the Astronomical Netherlands Satellite, and consists of no more than 5 points only, respectively at the central wavelengths of 1550, 1800, 2200, 2500, and $3300 \AA$, simply because the instrument onboard the satellite had only 5 channels. One can question the reliability of such measurement given the extremely low resolution, and the imperfect fit used to derive the color excess.

Interestingly, McClintock and Remillard (2000) published a HST/STIS spectrum of A0620-00 ranging from 1900 to $3100 \AA$, clearly revealing the absence of a "feature" at $2200 \AA$ (see their Fig. 1, upper panel). This absence of any strong extinction feature in the UV spectrum of McClintock and Remillard (2000) (who cite Barret et al. 1996, for the distance) seems to show that the extinction might be particularly low. This is consistent with a location of A0620-00 far from the

\footnotetext{
${ }^{11}$ We obviously ignore here the possible problems with the determinations of all other microquasars listed in Jonker and Nelemans (2004), and in particular XTE J1118+480 which has a published distance of $D=1.8 \pm 0.6 \mathrm{kpc}$.
}

Galactic plane $\left(\mathrm{l}=209.96^{\circ}, \mathrm{b}=6.54^{\circ}\right.$; R.A. $=6^{h} 22^{m} 44.4^{s}$, Dec. $=-00^{\circ} 20^{m} 45^{s}$ ).

More recently, Gelino et al. (2001) determine a distance of $1164 \pm 114$ pc for A0620-00 using the color excess of $\mathrm{Wu}$ et al. (1976). This distance has also been used by Shahbaz et al. (2004). Gallo et al. (2006) still use the older value of $1.2 \pm 0.4 \mathrm{kpc}$ citing Shahbaz et al. (1994), Gelino et al. (2001) and Jonker and Nelemans (2004). Finally, we note that Pal and Chakrabarti (2005) still use the distance range of Shahbaz et al. (1994), and that Esin et al. (2000, who obtained $D=1.4 \mathrm{kpc}$ ) cite Shahbaz et al. (1994) and Barret et al. (1996). All these studies, even those being very recent, directly rely on the 30-years old determination of $E(B-V)$ by Wu et al. (1976), even if they do not cite the original paper.

\subsection{A wealth of uncertain estimations}

Barret et al. (1996) use two different methods to estimate the distance of A0620-00, and found a value $(1.2 \mathrm{kpc})$ "in agreement with previous determinations". We note that they do not apply either method to GRO J1655-40 (which is also discussed in the paper) for which they take the literature value, $3.2 \mathrm{kpc}$.

The first method is the magnitude-comparison method (equation 2), assuming that the Roche-lobe radius derived from dynamical studies is equal to the "radius" of the secondary star. Moreover, Barret and coworkers state that the absolute magnitude depends only on the spectral type. Using another method to compute the Roche radius requiring a mass assumption ( $M=0.4 M_{\odot}$ for mid-K secondaries), Barret and collaborators confirm their distance value: $D=1.2 \mathrm{kpc}$. No uncertainties are provided, but they estimate them at about 25\%. They also cite Haswell et al. (1993) and Shahbaz et al. (1994), who provide the optical derredened magnitude and hence rely on the non-existent feature measured by Wu et al. (1976).

Oke and Greenstein (1977), cited by Jonker and Nelemans (2004) on their Table 3. discussed in Sec. 4, estimate the color excess using simultaneously the relations of Spitzer (1948, $E(B-V)=0.44$ with a large uncertainty), Wampler (1966, $E(B-V)$ ranging from 0.25 to 0.6) and Aannestad and Purcell (1973). But Spitzer use the data of Stebbins et al. (1940), and Aannestad and coworkers use that of York (1971). Oke and Greenstein (1977) conclude that "these methods cannot rule out much larger distances, since the object is largely out of the galactic plane". Finally, they use a distance of $870 \mathrm{pc}$ using another estimation of the absolute magnitude of the secondary dwarf star, and claim that this new value is "somewhat smaller than previous estimates [...] but not inconsistent with them". 
To summarize, it appears that all methods are more or less giving about the same result around $1 \mathrm{kpc}$, but with still a rather large scatter around this value. We also note that most estimations rely on the determination of the color excess by Wu et al.(1976), even in most recent literature.

Below, we use VLT/UVES spectra to apply the maximal-distance method of Foellmi et al. (2006) which, even systematically uncertain, provides an model-independent estimation of the distance. Interestingly, we can measure the equivalent width of the sodium doublet in these UVES spectra of A0620-00: $E W=0.5 \pm 0.1 \AA$. The value is slightly outside the allowed range given in Sec. 3.1, and more importantly, equation 11 might simply not be applicable to an object outside the galactic plane, in the direction of the anticenter, since it is outside the directions where the relationship has been calibrated. If we use it nonetheless with caution, we obtain $E(B-V)=0.22 \pm 0.07$. Assuming $R=3.1$, we have $A_{V}=0.68$. According to Shahbaz et al. (1994), the secondary star radius is $0.8 R_{\odot}$, its temperature $4000 \mathrm{~K}$ (implying a luminosity of $L=0.14 L_{\odot}$, that is an absolute magnitude of $M_{V}=7.0$ ), its visual magnitude $V=18.2$ and $40 \%$ disc contamination (i.e. a magnitude increase of $0.55 \mathrm{mag}$ ). The corresponding distance is $1.3 \mathrm{kpc}$, and 1.6 without veiling.

Could actually A0620-00 be further away? Given the various uncertainties, especially on the secondary star radius, this distance is certainly not conclusive.

\subsection{Astrometric estimations of the proper motion}

We looked in public archives for images that could provide an astrometrical estimation of the distance of A0620-00. Unfortunately, it has not been observed with an imaging technique by $H S T$. We were finally able to find only acquisition images in the ESO archive with the following instruments: $3.6 \mathrm{~m} / \mathrm{EFOSC} 2$ (ESO, La Silla Observatory), VLT/ISAAC and VLT/FORS1 (ESO, Cerro Paranal Observatory). Unfortunately, ISAAC frames proved to be useless because of the too small number of stars in the field of view, and were discarded. Finally, we also retrieved SuperCOSMOS images, which have a much poorer pixel scale but provide the largest baseline in time. Table 2 summarize the properties of the three datasets.

In order to check if the star has been ejected from a cluster with a runaway velocity, similarly to GRO J1655-40, we performed astrometrical calculations with the above archives images. We chose the EFOSC2 image to be the reference since it had the sharpest PSF, and selected 40 bright isolated stars that were visible on all other frames. We computed the geometrical transformation map between the reference and the other images using the fitted positions of these 40 stars. Finally, we computed the expected position of the target on the EFOSC2 image for a given transformation matrix and its actual position on this image. The difference in pixels was transformed into arcsecond using the pixel scale of the EFOSC2 CCD. Finally, the results were divided by the time elapsed between the reference and the given image.

It appeared that the best transformation fit was obtained between the FORS1 and EFOSC2 images, with an rms $=0.1508$ and 0.1506 pixels in the $\mathrm{X}$ and $\mathrm{Y}$ direction respectively. This translates into an uncertainty of about 30 mas. The very small observed difference of the position of A0620-00 between the FORS1 and EFOSC2 images was clearly below this value: -10.4 and 9.0 mas in the $\mathrm{X}$ and $\mathrm{Y}$ directions respectively. For the SuperCOSMOS images, the rms achieved in the transformation fit is 0.65 and 0.20 pixels, corresponding to a uncertainty of 436 and 134 mas respectively. The observed displacement was again inside the uncertainties of 71 and 136 mas.

Taking the 2.03 years difference between the FORS1 and EFOSC2 images, we obtain a maximum proper motion of about 15-20 mas/yr, which roughly corresponds to that obtained with SuperCOSMOS images that are 22.08 years apart from EFOSC2 ones. It is possible to look at the possible presence of a cluster of stars within a given radius, taking into account this upper limit of the astrometric motion of A0620-00 on the sky. Looking at a region of radius of about 3 degrees using SIMBAD, we found two clusters: [KPR2005] 22 (Kharchenko et al., 2005), and C 0619+023 (Collinder, 1931). While there is no information on the latter, the former has an estimated distance of $1.5 \mathrm{kpc}$. It is located 2.2808 degrees away from A0620-00, corresponding to a projected separation on the sky of $\sim 59.7 \mathrm{pc}$ at $1.5 \mathrm{kpc}$. Given the upper limit of the projected proper motion of A0620-00, one can estimate its minimal age to be about 367000 years.

The current data does not allow us to derive strong constraints on the proper motion of A0620-00, and therefore on its possible origin. Combined with the systemic radial velocity (4 $\mathrm{km} \mathrm{s}^{-1}$ according to Marsh et al. 1994), it would be interesting to simulate possible galactic orbits for A0620-00, similarly to what Mirabel et al. (2002) have done for GRO J1655-40. 
Table 2: Summary of the archival data used to derive astrometry of A0620-00. The instrument/project, observatory location, date of observations and bandpass (filters) are indicated as well as the Field-of-View in arcminutes, and the pixel scale (in arcseconds per pixel). The pixel scale of SuperCOSMOS plates are computed given a plate scale of 67.14 '/mm and $10 \mu m$ pixel size. The FOV of SuperCOSMOS images is originally $6.4 \times 6.4$ degrees, but smaller regions can be retrieved electronically.

\begin{tabular}{cccccc}
\hline Instrument & Location & Date & Filter (name) & FOV ('x') & Scale ('/pixel) \\
\hline SuperCOSMOS/UK Schmidt & Siding Spring Obs. & $16 / 02 / 1983$ & I (RG715) & up to 384x384 & 0.6714 \\
VLT/FORS1 & VLT/Paranal & $7 / 01 / 2003$ & V (V_BESS+35) & $6.8 \times 6.8$ & 0.200 \\
La Silla/EFOSC2 & La Silla (3.6m) & $16-18 / 01 / 2005$ & V (V\#641) & $5.3 \times 5.3$ & $0.31(2 \times 2$ bin) \\
\hline
\end{tabular}

\section{The maximal-distance method of Foellmi et al. (2006) and its application to A0620-00}

\subsection{Issues in the method}

In Foellmi et al. (2006) a new method allowing to estimate a maximum distance has been presented and applied to the case of GRO J1655-40. It is based on the comparison between the calibrated spectroscopic fluxes of the secondary star and that of a companion star of similar spectral type and luminosity class which needs to be close enough to have a negligible absorption. The comparison is made with spectra obtained with the same instrument (VLT/UVES) configured with an identical setup. The main issue with this method is that one needs to make an hypothesis about the absolute magnitude difference between the two stars. More precisely, if we call $f$ the ratio of the spectroscopic fluxes between the secondary star in the microquasar, and the comparison star, Foellmi et al. (2006) derive the following relation:

$$
a=5 \log \left(\frac{D_{2}}{D_{1}} \frac{1}{\sqrt{f}}\right)+M_{2}-M_{1} \geq 0
$$

where $a$ is the (spectroscopic) absorption toward the target (i.e. the average absorption within the narrow wavelength range given by the UVES spectra used to make the comparison). $D_{1}$ and $D_{2}$ are the distances of the microquasar and the reference star respectively. Obviously, $a$ must be null or positive. The difference between the two absolute magnitudes $M_{1}$ and $M_{2}$ is however impossible to evaluate. Or, in other wods, how different is the absolute magnitude $M_{1}$ compared to that of the reference star, given that the spectra are directly compared. For GRO J1655-40, Foellmi et al. (2006) allowed for a large range and made the hypothesis that $M_{1}$ is comprised between $M_{2}-1$ and $M_{2}+1$. They finally found that $D \lesssim 1.7 \mathrm{kpc}$ by comparing flux of GRO J1655-40 to that of 4 nearby stars of similar spectral types. However, because the absolute magnitude is unknown, this method has a systematic intrinsic uncertainty.

Since then, various authors mention this distance revision (Caballero-García et al. 2006, Takahashi et al.
2008; Joinet et al., 2008), but simply continue to use the canonical value. Sala et al. (2007) used XMM-Newton data to derive a connection between the inner radius of the accretion disk and the distance. They argue that if the distance is less than $1.7 \mathrm{kpc}$ and its mass is less than $5 M_{\odot}$, then the inner accretion disk radius will be inside the gravitational radius of the black hole. However, as noted by Combi et al. (2007), the mass and spin of the black hole are uncertain. If the black hole were rapidly rotating as suggested by McClintock et al. (2006) and its mass less than $5 M_{\odot}$, the horizon of the Kerr black hole could then be well inside the inner accretion radius. Furthermore, Sala et al. (2007) admit that an estimate of the inner accretion radius using $X M M$ data is model dependent.

Lasota (2008) mention that if we were to accept the new and smaller distance, the secondary in GRO J165540 would not be filling its Roche-lobe (a similar remark is made in Caballero-García et al. 2007, who do not provide however any reference about it) and in this case too we would have to invoke a mass transfer instability to explain the outbursts. But we have seen above that a complete modelling of the multi-color lightcurves with a good understanding of the disk emission and the absorption is still lacking.

\subsection{Application to A0620-00}

In order to check the distance of A0620-00, we applied two different methods. The first one is that of the red clump giant stars. Unfortunately, it proved to be useless because of the too small number of stars in the 2MASS database in the direction of A0620-00, even taking a box of one degree around it. We were simply unable to identify a Red Clump Giant star branch in the CMD. It can certainly be explained by the direction of A0620-00 in the sky, which points away from the galactic center. The other method is that of Foellmi et al. (2006). To complement the study, we derive constraints on its proper motion using archival imaging data.

We looked for VLT-UVES archival data on A0620 00, with the aim at comparing them to the UVES spectra 


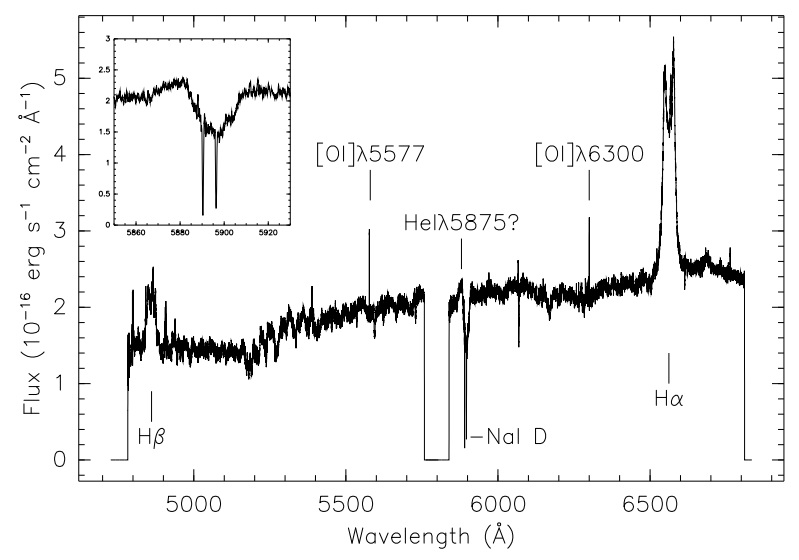

Figure 6: UVES spectrum of A0620-00 (lower and upper CCDs combined), smoothed with a boxcar of 11 pixels. Major spectral features are marked. The inset shows a close-up of the sodium doublet region. Neilsen et al. (2008) identify with caution the possible presence of $\mathrm{HeI} \lambda 5875$ in this region.

of the UVESPOP database $e^{12}$ (see Bagnulo et al. 2003). We have found 20 spectra of the target (ESO program ID 066.D-0157(A), P.I. Maeder), split in two different wavelength range: 4790 to $5755 \AA$, and 5840 to $6805 \AA$. The spectra were taken in 2000, December 5 (12 spectra), 17 ( 2 spectra) and 21 (6 spectra). The have been reduced and calibrated in exactly the same way as for GRO J1655-40 (see Foellmi et al. 2006, to which the reader is referred for a detailed description of the reduction and flux-calibration of the spectra). The average spectrum is shown in Fig. 6. Moreover, we also retrieved from the UVESPOP the spectra of three different single stars with similar spectral types and luminosity classes: HD 10361 (K5V), HD 100623 (K0V) and HD 209100 (K4.5V).

As in Foellmi et al. (2006), we compare the fluxcalibrated spectrum of A0620-00 with that of the comparison stars (ignoring the $\mathrm{H} \alpha$ line, in emission in A0620-00 spectrum). The results are shown in Fig. 7 and summarized in Table 3 Given that $a$ must be null or positive, we can see that the resulting maximum distance is significantly smaller than $1 \mathrm{kpc}$, with a mean around $0.4 \mathrm{kpc}$. We emphasize here that we accounted for $40 \%$ veiling of the disk (i.e. we multiplied the $f$ ratios in the table by a factor 0.6 ) before computing the distance. Any smaller fraction of the contamination will bring the object even closer to the Sun.

${ }^{12}$ http://www.sc.eso.org/santiago/uvespop/

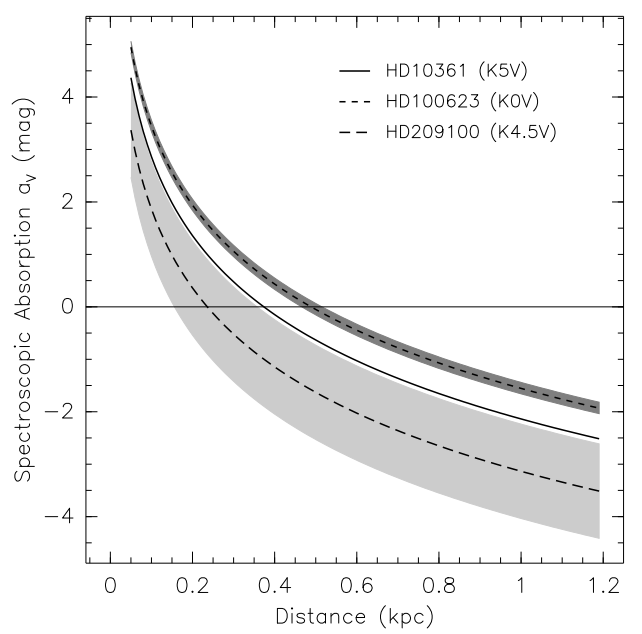

Figure 7: Relation between the spectroscopic absorption $a$ and the distance, following the equation 17 The condition that the absorption must be positive is represented by the horizontal line. The light gray area shows the systematic uncertainty of \pm 1 magnitude, while the dark gray area shows an uncertainty of $10 \%$ on the spectroscopic flux. It shows that the likely distance of A0620-00 is probably smaller than $0.5 \mathrm{kpc}$.

\section{Summary \& Conclusions}

It has been clearly shown that despite a large number of variants in distance methods, it is of prime importance to be extremely rigorous with assumptions and with the use of results made earlier and usually by others. The distance of microquasars, and in general of compact objects in our Galaxy, will benefit in a few years of the results of the satellite Gaia. Until then, we will certainly have to combine various types of information to infer good estimates to the distance of these objects. We mention here the work of Lazorenko et al. (2007) who have obtained, through astrometric measurements using VLT/FORS1, a precision of 30 microarcseconds, similarly to what is expected for the VLTI instrument PRIMA, currently being commissioned at the ESO Cerro Paranal Observatory. This method requires a rather simple observational setup, a large field, and a large number of stars in the field. These requirements are easily fulfilled in the case of microquasars in the galactic plane like GRO J1655-40, although it might again be more difficult or impossible for A0620-00.

From the present work, we can conclude that:

- The use of the color excess measured using the sodium lines is risky. Not only one must have a spectroscopic resolution high enough, but its applicability range is limited. Moreover, one must 
Table 3: The three dwarf $\mathrm{K}$ stars used to compute the maximum distance of A0620-00 are summarized. The star's name, spectral type, distance (computed from the HIPPARCOS parallax), absolute magnitude, $(B-V)$ color (obtained from SIMBAD) and the ratio of the flux-calibrated spectrum of A0620-00 with that of the star are indicated. The last column indicates the ranges of maximum distance $D$ of A0620-00 obtained through the constraint of $a \geq 0$ with the two limits of the absolute magnitude $M_{1}$. See text for details. The quoted $f$ flux ratios are multiplied by a factor 0.6 before estimating the distance to account for a contamination of the accretion disk of $40 \%$ according to Shahbaz et al. (1994). The uncertainty on the distance values is $\pm 0.2 \mathrm{kpc}$. The error on the absolute magnitude $M_{2}$ is computed from the error on the HIPPARCOS distance.

\begin{tabular}{llccccc}
\hline Star & Sp. Type & $\begin{array}{c}\text { Distance } \\
(\mathrm{pc})\end{array}$ & $\begin{array}{c}M_{2} \\
(\mathrm{mag})\end{array}$ & $\begin{array}{c}B-V \\
(\mathrm{mag})\end{array}$ & $f$ & $\begin{array}{l}\text { Max. } D_{1} \text { (spec) } \\
(\mathrm{kpc})\end{array}$ \\
\hline HD 10361 & K5V & $6.6 \pm 0.1$ & $6.7 \pm 0.2$ & 0.85 & $(5.2 \pm 0.9) 10^{-4}$ & $0.24-0.59$ \\
HD 100623 & K0V & $9.5 \pm 0.1$ & $6.1 \pm 0.1$ & 0.81 & $(6.3 \pm 0.9) 10^{-4}$ & $0.31-0.77$ \\
HD 209100 & K4.5V & $3.61 \pm 0.05$ & $6.9 \pm 0.2$ & 1.06 & $(3.9 \pm 0.9) 10^{-4}$ & $0.15-0.37$ \\
\hline
\end{tabular}

be careful when choosing the relationship between the equivalent width and the color excess.

- There is no proven systematic overestimation of the optical absorption as determined from X-ray data compared to that inferred from optical data.

- The optical data also needs to be checked for being taken truly during optical quiescence or not. This is crucial for lightcurve models.

- The quality of the data and the uncertainties associated to it must be assessed rigorously. It is meaningless to apply a sophisticated model on data of limited quality.

- The maximum-distance method proposed by Foellmi et al. (2006) contains a systematic uncertainty, although its main hypothesis is sometimes implicitly used by others.

\section{About GRO J1655-40:}

- We have shown that although the upper limit of $3.5 \mathrm{kpc}$ is a rather firm measurement, the value of $3.2 \mathrm{kpc}$ has never been really measured.

- We have also shown that the lower limit of $3.0 \mathrm{kpc}$ is questionable and actually based on a questionable assumption on the interpretation of absorption lines in the radio spectrum. Moreover, we have challenged the relevance of comparing two radio spectra in a region where there $\mathrm{HI}$ clouds with anomalous velocities.

- We have estimated that $E(B-V) \sim 1.0$ and $A_{V} \sim$ 3.49 .

- The peculiar absorption determined from X-rays towards GRO J1655-40 possibly indicate the presence of local dust close to the object. However, the $\mathrm{X}$-ray flux in quiescence is also very variable.
- The studies of the orbit and secondary star of GRO J1655-40 rely very much on the quality of the model of the disk, and on the assumed fixed parameters, often extracted from other incomplete studies. As matter of fact, no true model of the lightcurves of GRO J1655-40 combining the radial-velocity measurement and the multi-color lightcurves has been made while letting all the parameters truly vary at the same time.

- A new distance method using red clump giant stars applied to GRO J1655-40 however confirms a distance less than $2 \mathrm{kpc}$.

- Although not proven, the new estimation of a smaller distance of GRO J1655-40 strengthen the idea of its possible origin in NGC 6242. It would make GRO J1655-40 one of the closest black holes to the Sun.

- At $1.0 \mathrm{kpc}, \beta=0.28$ and there would be no misalignment between the disk and the jets, since $\theta \sim 71^{\circ}$

As for A0620-00:

- We have shown that the published distance and many confirmations of it are based (not always explicitely) on a single measurement of the color excess made 30 years ago on a spectrum made of 5 points, which is moreover in contradiction with an HST/STIS spectrum.

- We have applied the maximum-distance method of Foellmi et al. (2006) to A0620-00 and found that it could be indeed located much closer, to a distance of $\sim 0.4 \mathrm{kpc}$.

- The example of A0620-00 illustrate that normal distance methods based on comparing the apparent and absolute magnitudes are difficult to apply to A0620-00 since the estimation of the extinction 
cannot rely on relationship established in the galactic plane. For this reason also, the red clump giant star distance method could not be applied to A0620-00.

- We have also used archival images to infer an upper limit of the proper motion of A0620-00, which appears quite small. Although we have found a cluster of stars 2.8 degrees away, the origin of A0620-00 remains uncertain.

Finally, to the question "What is the closest black hole to the Sun?", our answer is the black hole X-ray binary A0620-00.

\section{Acknowledgments}

C.F. warmly acknowledges T.H. Dall and E. Depagne for advices and a critical reading of the manuscript. C.F. wants to thank G. Nelemans for critical discussions on the details of some distance methods, E. Moraux for help with the red clump stars, and F. Comeron for giving the necessary last bit of motivation to complete such study. C.F. is indebted to A. Burgess for proofreading the English grammar and orthography of the manuscript. C.F. thanks the anonymous referee for corrections and pointing out an error on A0620-00, and C. Sterken for insightful discussions about scientific rigor. This research has made a really extensive use of the European Southern Observatory archive, NASA's Astrophysics Data System, the arXiv astro-ph, and the Central Bureau for Astronomical Telegrams of the IAU. This research has made use of the SIMBAD database, operated at CDS, Strasbourg, France. C.F. acknowledges the Institut de Radio-Astronomie Milimetrique (IRAM) in Grenoble for keeping old volumes of Nature in its library. C.F. also acknowledges the use of SuperCOSMOS Sky Surveys. Finally, C.F. acknowledges support from the Swiss National Science Foundation (grant no PA0022-115328).

\section{A. Bibliographical glitches}

We mention here the few bibliographic issues that we have encountered in studying the question of the distance of GRO J1655-40 and A0620-00. The Nature paper by Bailyn et al. (1995b) quote Arnett \& Bowers in 1978 about the maximum neutron star mass, while the correct year is 1977 (Arnett and Bowers, 1977). Phillips et al. (1999) quote "Bailyn et al. (1996b)" from which they use the outburst data. However the correct year is 1995. Trimble and Leonard (1995) mention the IAU Circular nb. 6063 of Inoue et al. (1994) but attribute it for some reasons to Reynolds \& Jauncy. When looking for the X-ray fluxes of GRO J1655-40 in Garcia et al. (1998) quoted by Mirabel et al. (2002), the authors referred to Barret et al. (1996). However, the value of the X-ray flux is actually quoted from another paper, said to be "in press", by Zhang et al. in 1996, in the Astronomy \& Astrophysics Supplement Series. There are three publications that could match this reference: Zhang et al. (1996a) that is describing X-ray BATSE/CGRO observations through Earth occultation and where GRO J1655-40 is not mentioned, Zhang et al. (1996b) that is about GRO J1849-03 only and Zhang et al. (1996c), in which only the source $4 \mathrm{U} 1608-522$ is discussed. Moreover, there is no publications with Zhang as a first author in 1997 and in A\&AS. There is actually a paper in 1997 in ApJ:Zhang et al. (1997) where ASCA and BATSE X-ray fluxes of GRO J1655-40 are discussed, and this latter paper is certainly the correct reference. As for the color excess coming from other sources for GS 1124-684 cited by Greiner et al. (1994a), there is a reference "West et al. (1991)". But in the reference list, we find only an IAU Circular with only "West R.M." as author, and West, R.M. Della Valle M., Jarvis B., 1991, in "Workshop on Nova Muscae 1991", Lyngby, May 1991. Interestingly, the NASA ADS system does not list any of those two references. The only seemingly reference is the IAU Circular West et al. (1991, no 5165) but with the additional and final coauthor Pizzaro G. not mentioned in the item of the reference list of Greiner et al. (1994a). Finally, Orosz and Bailyn (1997) refer to a submitted paper by Robinson C. et al. (1996) in the Astrophysical Journal, that cannot actually be found in NASA ADS. We were actually not able to find any paper by C. Robinson as a first, second or third author on this subject, in 1995, 1996 or later.

\section{References}

Aannestad, P. A., Purcell, E. M., Jan 1973. Interstellar grains. ARA\&A, 11, 309.

Allen, C. W., 1973. Astrophysical Quantities (Third Edition).

Arnett, W. D., Bowers, R. L., 1977. A microscopic interpretation of neutron star structure. ApJS, 33, 415.

Asai, K., Dotani, T., Hoshi, R., Tanaka, Y., Robinson, C. R., Terada, K., Dec 1998. Asca observations of transient X-ray sources in quiescence. PASJ, 50, 611.

Bagnulo, S., Jehin, E., Ledoux, C., Cabanac, R., Melo, C., Gilmozzi, R., Team, T. E. P. S. O., 2003. The uves paranal observatory project: A library of high- resolution spectra of stars across the hertzsprung-russell diagram. The Messenger, 114, 10-14.

Bailey, J., 1981. The distances of cataclysmic variables. MNRAS, 197, 31-39.

Bailyn, C. D., Orosz, J. A., Girard, T. M., Jogee, S., della Valle, M., Begam, M. C., Fruchter, A. S., Gonzalez, R., Ianna, P. A., Layden, 
A. C., Martins, D. H., Smith, M., 1995a. The optical counterpart of the superluminal source GRO J1655-40. Nature, 374, 701.

Bailyn, C. D., Orosz, J. A., McClintock, J. E., Remillard, R. A., 1995b. Dynamical evidence for a black hole in the eclipsing X-ray nova GRO J1655-40. Nature, 378, 157.

Barbon, R., Benetti, S., Rosino, L., Cappellaro, E., Turatto, M., 1990. Type ia supernova 1989b in ngc 3627. A\&A, 237, 79-90.

Barret, D., McClintock, J. E., Grindlay, J. E., Dec 1996. Luminosity differences between black holes and neutron stars. ApJ, 473, 963.

Beer, M. E., Podsiadlowski, P., Mar 2002. The quiescent light curve and the evolutionary state of GRO J1655-40. MNRAS, 331, 351.

Bianchini, A., della Valle, M., Masetti, N., Margoni, R., 1997. Spectroscopic study of GRO J1655-40: the outburst and the decline. A\&A, 321, 477-484.

Bradt, H. V. D., McClintock, J. E., 1983. The optical counterparts of compact galactic X-ray sources. ARA\&A, 21, 13-66.

Brandt, W. N., Podsiadlowski, P., Sigurdsson, S., Nov 1995. On the high space velocity of X-ray nova sco 1994: implications for the formation of its black hole. MNRAS, 277, L35.

Brocksopp, C., McGowan, K. E., Krimm, H., Godet, O., Roming, P., Mason, K. O., Gehrels, N., Still, M., Page, K. L., Moretti, A., Shrader, C. R., Campana, S., Kennea, J., 2006. The 2005 outburst of GRO J1655-40: spectral evolution of the rise, as observed by swift. MNRAS, 365, 1203-1214.

Buxton, M. M., Vennes, S., 2001. Atmospheric modelling of the companion star in GRO J1655-40. Publications of the Astronomical Society of Australia, 18, 91-97.

Caballero-Garcia, M., Kuulkers, E., Kretschmar, P., Domingo, A., Miller, J. M., Mas-Hesse, J. M., 2006. ToO observations of GRO J1655-40 in outburst with INTEGRAL. arXiv:astroph/0609491

Caballero-García, M.D., Miller, J.M., Kuulkers, E., Díaz Trigo, M. Homan, J., Lewin, W.H.G., Kretschmar, P., Domingo, A., MasHesse, J.M., Wijnands, R., Fabian, A.C., Fender, R.P., van der Klis, M., 2007. The High-Energy Emission of GRO J1655-40 As Revealed with INTEGRAL Spectroscopy of the 2005 Outburst. ApJ, 669, 534.

Cantrell, A. G., Bailyn, C. D., McClintock, J. E., Orosz, J.A., 2008. Optical State Changes in the X-Ray-quiescent Black Hole A062000., ApJ, 673, L159.

Caswell, J. L., Murray, J. D., Roger, R. S., Cole, D. J., Cooke, D. J., Dec 1975. Neutral hydrogen absorption measurements yielding kinematic distances for 42 continuum sources in the galactic plane. A\&A, 45, 239.

Chakrabarti, S.K., Debnath, D., Nandi, A., Pal, P.S., 2008. Evolution of the quasi-periodic oscillation frequency in GRO J1655-40 - Implications for accretion disk dynamics. A\&A, 489, L41.

Cheng, F. H., Horne, K., Panagia, N., Shrader, C. R., Gilmozzi, R. Paresce, F., Lund, N., Oct 1992. The hubble space telescope observations of X-ray nova muscae 1991 and its spectral evolution. ApJ, 397, 664.

Chevalier, C., Ilovaisky, S. A., 1990. Optical studies of transient lowmass X-ray binaries. iii - photometry of gs $2000+25$ during decay and quiescence. A\&A, 238, 163-169.

Clark, J. S., Charles, P. A., Clarkson, W. I., Coe, M. J., 2003. Near ir spectroscopy of the X-ray binary circinus X-1. A\&A, 400, 655658

Cohen, J. G., 1975. Optical interstellar lines in southern supergiants. ApJ, 197, 117-122.

Collinder, P., 1931. On structured properties of open galactic clusters and their spatial distribution. Annals of the Observatory of Lund, 2,1

Combi, J. A., Bronfman, L., Mirabel, I. F., May 2007. New evidence on the origin of the microquasar GRO J1655-40. A\&A, 467, 597.

Combi, J. A., Romero, G. E., Benaglia, P., Mirabel, I. F., 2001. The radio surroundings of the microquasar GRO J1655-40. A\&A, 370, L5-L8.

Crawford, D. L., Mandwewala, N., Dec 1976. Interstellar reddening relations in the ubv, uvby, and geneva systems. PASP, 88, 917.

Crawford, I. A., Barlow, M. J., Blades, J. C., Jan 1989. Highresolution observations of interstellar na $\mathrm{i}$ and ca ii absorption lines toward the scorpius ob1 association. Astrophysical Journal, 336, 212.

dal Fiume, D., Frontera, F., Orlandini, M., Amati, L., del Sordo, S., Stella, L., Belloni, T. M., Ricci, D., Capalbi, M., Daniele, M. R., 1999. Xte j1859+226. IAU Circular 7291, 2.

della Valle, M., 1994. X-ray nova in scorpius. IAU Circular 6052.

della Valle, M., Duerbeck, H. W., 1993. Study of nova shells - part one - v1229-aquilae - nova 1970 - nebular expansion parallax and luminosity. A\&A, 275, 239.

della Valle, M., Jarvis, B. J., West, R. M., 1991. Evidence for a black hole in the X-ray nova muscae 1991. Nature, 353, 50-52.

Dubus, G., Lasota, J.-P., Hameury, J.-M., Charles, P., Feb 1999. X-ray irradiation in low-mass binary systems. MNRAS, 303, 139

Durant, M., van Kerkwijk, M. H., Distances to anomalous X-ray pulsars using red clump stars. ApJ, 650, 1070.

Eggleton, P. P., 1983. Approximations to the radii of roche lobes. ApJ, 268,368

Esin, A. A., Kuulkers, E., McClintock, J. E., Narayan, R., 2000. Optical light curves of the black hole binaries GRS 1124-68 and A0620-00 in outburst: The importance of irradiation. ApJ, 532, 1069.

Fitzgerald, M. P., 1970. The intrinsic colours of stars and two-colour reddening lines. A\&A, 4, 234.

Fitzpatrick, E. L., Jan 1999. Correcting for the effects of interstellar extinction. The Publications of the Astronomical Society of the Pacific 111,63 .

Foellmi, C., 2006. Proceedings of the VI Microquasar Workshop: Microquasars and Beyond, 62.

Foellmi, C., 2007. What really is the relativistic radio-jet distance of the galactic microquasar GRO J1655-40? The Future of Photometric, Spectrophotometric and Polarimetric Standardization, ASP Conference Series, 364, 597.

Foellmi, C., Dall, T. H, Depagne, E., 2007. On the abundances of GRO J1655-40. A\&A, 464, L61-L64.

Foellmi, C., Depagne, E., Dall, T. H., Mirabel, I. F., 2006. On the distance of GRO J1655-40. A\&A, 457, 249-255.

Gallo, E., Fender, R. P., Miller-Jones, J. C. A., Merloni, A., Jonker, P. G., Heinz, S., Maccarone, T. J., van der Klis, M., Aug 2006. A radio-emitting outflow in the quiescent state of A0620-00: implications for modelling low-luminosity black hole binaries. MNRAS, 370,1351 .

Garcia, M. R., McClintock, J. E., Narayan, R., Callanan, P. J., Jan 1998. Black hole event horizons and X-ray nova luminosities - update. Wild Stars In The Old West: Proceedings of the 13th North American Workshop on Cataclysmic Variables and Related Objects. ASP Conference Series, 137, 506.

Garcia, M. R., McClintock, J. E., Narayan, R., Callanan, P. J., Barret, D., Murray, S. S., 2001. New evidence for black hole event horizons from Chandra. ApJ, 553, L47-L50.

Gelino, D. M., Harrison, T. E., Orosz, J. A., Nov 2001. A multiwavelength, multiepoch study of the soft X-ray transient prototype, V616 Monocerotis (A0620-00). AJ, 122, 2668.

Gierliński, M., Maciołek-Niedźwiecki, A., Ebisawa, K., 2001. Application of a relativistic accretion disc model to X-ray spectra of LMC X-1 and GRO J1655-40. MNRAS, 325, 1253-1265.

Gonzalez-Riestra, R., Clavel, J., Cassatella, A., Krautter, J., Gilmore, A. C., 1991. Nova in the large magellanic cloud, IAU Circu$\operatorname{lar} 5253$.

González Hernández, J. I., Rebolo, R., Israelian, G., 2008. The Black 
Hole Binary Nova Scorpii 1994 (GRO J1655-40): An improved chemical analysis. A\&A, 478, 203.

Gottlieb, D. M., Upson, W. L., Aug 1969. Local interstellar reddening. ApJ, 157, 611.

Gray, D. F., 1992. The Observation and Analysis of Stellar Photospheres, Cambridge University Press.

Greene, J., Bailyn, C. D., Orosz, J. A., 2001. Optical and infrared photometry of the microquasar GRO J1655-40 in quiescence. ApJ, 554, 1290-1297.

Greiner, J., Hasinger, G., Molendi, S., Ebisawa, K., 1994a. Nearsimultaneous ROSAT and Ginga observations of the 1991 X-ray transient in Musca. A\&A, 285, 509.

Greiner, J., Predehl, P., Harmon, B. A., 1994b. ROSAT observation of the 4 U 1543-47 outburst in 1992. AIP Conf. Series 304, 314-318.

Greiner, J., Predehl, P., Pohl, M., 1995. ROSAT observations of GRO J1655-40. A\&A, 297, L67.

Grindlay, J. E., Hertz, P., 1981. Discovery of an obscured globular cluster associated with GX 354+0 (=4U/MXB 1728-34). ApJ, 247, L17-L21.

Groenewegen, M. A. T., Udalski, A., Bono, G., Apr 2008. The distance to the galactic centre based on population II cepheids and RR Lyrae stars. A\&A, 481, 441-448.

Guver, T., Ozel, F., Cabrera-Lavers, A., Wroblewski, P., 2008. The distance, mass, and radius of the neutron star in $4 \mathrm{U}$ 1608-52. ApJ submitted, arXiv:0811.3979

Harmon, B. A., Wilson, C. A., Zhang, S. N., Paciesas, W. S., Fishman, G. J., Hjellming, R. M., Rupen, M. P., Scott, D. M., Briggs, M. S., Rubin, B. C., 1995. Correlations between X-ray outbursts and relativistic ejections in the X-ray transient GRO J1655-40. Nature, $374,703$.

Haswell, C. A., Robinson, E. L., Horne, K., Stiening, R. F., Abbott, T M. C., 1993. On the mass of the compact object in the black hole binary A0620-00. ApJ, 411, 802.

Herbig, G. H., 1975. The diffuse interstellar bands. iv - the region 4400-6850 a. ApJ, 196, 129

Hjellming, R., Johnston, K., Mar 1981a. Structure, strength, and polarization changes in radio source ss433. Nature, 290 (5802), 100107, 10.1038/290100a0.

Hjellming, R. M., Johnston, K. J., 1981b. An analysis of the proper motions of SS 433 radio jets. ApJ, 246, L141.

Hjellming, R. M., Johnston, K. J., 1988. Radio emission from conical jets associated with X-ray binaries. ApJ, 328, 600.

Hjellming, R. M., Rupen, M. P., 1995. Episodic ejection of relativistic jets by the X-ray transient GRO J1655-40. Nature, 375, 464.

Horne, K., Harlaftis, E. T., Baptista, R., Hellier, C., Allan, A., Johnston, H., Patterson, J., Kemp, J., Haswell, C., Chen, W., 1996. GRO J1655-40. IAU Circular 6406, 2.

Horne, K., Wade, R. A., Szkody, P., 1986, A dynamical model for the dwarf nova AH Herculis. MNRAS, 219, 791.

Hynes, R. I., Haswell, C. A., Chaty, S., Shrader, C. R., Cui, W., 2002. The evolving accretion disc in the black hole X-ray transient XTE J1859+226. MNRAS, 331, 169-179.

Hynes, R. I., Haswell, C. A., Shrader, C. R., Chen, W., Horne, K., Harlaftis, E. T., O’Brien, K., Hellier, C., Fender, R. P., 1998. The 1996 outburst of GRO J1655-40: the challenge of interpreting the multiwavelength spectra. MNRAS, 300, 64 .

Inoue, H., Nagase, F., Ishida, M., Sonobe, T., Ueda, Y., 1994. X-ray nova in scorpius. IAU Circular 6063.

Israelian, G., Rebolo, R., Basri, G., Casares, J., Martinìn, E. L., 1999. Evidence of a supernova origin for the black hole in the system GRO J1655-40. Nature, 401, 142.

Johnston, H. M., Wu, K., Fender, R. P., Cullen, J. G., 2001. Secular and orbital variability of Cir X-1 observed in optical spectra MNRAS, 328, 1193-1199.

Joinet, A., Kalemci, E., Senziani, F., 2008. Hard X-ray emission of the microquasar GRO J1655-40 during the rise of its 2005 outburst. ApJ, 679, 655.

Jonker, P. G., Nelemans, G., Oct 2004. The distances to galactic lowmass X-ray binaries: consequences for black hole luminosities and kicks. MNRAS, 354, 355

Kharchenko, N. V., Piskunov, A. E., Röser, S., Schilbach, E., Scholz, R.-D., 2005. Astrophysical parameters of galactic open clusters. A\&A, 438, 1163-1173.

Kobayashi, Y., Kubota, A., Nakazawa, K., Takahashi, T., Makishima, K., 2003. Observational evidence for a high-energy compton cloud in GRO J1655-40 under a high accretion rate. PASJ, 55, 273-279.

Kong, A. K. H., McClintock, J. E., Garcia, M. R., Murray, S. S., Barret, D., 2002. The X-ray spectra of black hole X-ray novae in quiescence as measured by Chandra. ApJ, 570, 277-286.

Koornneef, J., 1983. Near-infrared photometry. II - intrinsic colours and the absolute calibration from one to five micron. A\&A, 128, 84.

Kubota, A., Makishima, K., Ebisawa, K., 2001. Observational evidence for strong disk comptonization in GRO J1655-40. ApJ, 560, L147-L150.

Kuulkers, E., in't Zand, J. J. M., Cornelisse, R., Heise, J., Kong, A. K. H., Charles, P. A., Bazzano, A., Cocchi, M., Natalucci, L., Ubertini, P., 2000. Turmoil on the accretion disk of GRO J1655-40. A\&A, 358, 993-1000.

Lasota, J.-P., 2008. Adafs, accretion discs and outbursts in compact binaries. New Astronomy Reviews, 51, 752.

Lazorenko, P. F., Mayor, M., Dominik, M., Pepe, F., Segransan, D., Udry, S., 2007. High-precision astrometry on the VLT/FORS1 at time scales of few days. A\&A, 471, 1057-1067.

López-Corredoira, M., Cabrera-Lavers, A., Garzón, F., Hammersley, P. L., 2002. Old stellar galactic disc in near-plane regions according to 2mass: Scales, cut-off, flare and warp. A\&A, 394, 883.

Maccarone, T. J., 2002. On the misalignment of jets in microquasars. MNRAS, 336, 1371-1376.

Marsh, T. R., Robinson, E. L., Wood, J. H., 1994. Spectroscopy of A0620-00 - the Mass of the Black-Hole and an Image of its Accretion Disc. MNRAS, 266, 137.

Martin, R. G., Tout, C. A., Pringle, J. E., 2008. Alignment time-scale of the microquasar GRO J1655-40. MNRAS, 387, 188.

McCall, M. L., 2004. On determining extinction from reddening. AJ, 128, 2144.

McClintock, J. E., Remillard, R. A., Mar 2000. HST/STIS UV spectroscopy of two quiescent X-ray novae: A0620-00 and Centaurus X-4. ApJ, 531, 956.

McClintock, J. E., Shafee, R., Narayan, R., Remillard, R. A., Davis, S. W., Li, L.-X., 2006. The spin of the near-extreme Kerr black hole GRS 1915+105. ApJ, 652, 518-539.

McKay, D., Kesteven, M., 1994. X-ray nova in Scorpius. IAU Circular 6062.

Migliari, S., Tomsick, J.A., Markoff, S., Kalemci, E., Bailyn, C.D., Buxton, M., Corbel, S., Fender, R.P., Kaaret, P., 2007. Tracing the Jet Contribution to the Mid-IR over the 2005 Outburst of GRO J1655-40 via Broadband Spectral Modeling. ApJ, 670, 610.

Mignani, R. P., Luca, A. D., Caraveo, P. A., Mirabel, I. F., 2002. HST observations rule out the association between $\mathrm{Cir} \mathrm{x}-1$ and SNR G321.9-0.3. A\&A, 386, 487-491.

Miller, J. M., Raymond, J., Fabian, A. C., Steeghs, D., Homan, J., Reynolds, C., van der Klis, M., Wijnands, R., 2006. The magnetic nature of disk accretion onto black holes. Nature, 441, 953-955.

Mirabel, I. F., Oct 2004. Microquasar-AGN-GRB connections. Proceedings of the 5th INTEGRAL Workshop on the INTEGRAL Universe (ESA SP-552), 552, 175.

Mirabel, I. F., Mignani, R., Rodrigues, I., Combi, J. A., Rodriguez, L. F., Guglielmetti, F., 2002. The runaway black hole GRO J165540. A\&A, 395, 595-599. 
Mirabel, I. F., Rodriguez, L. F., 1994. A superluminal source in the galaxy. Nature, $371,46$.

Munari, U., Zwitter, T., 1997. Equivalent width of Na I and K I lines and reddening. A\&A, 318, 269-274.

Neilsen, J., Steeghs, D., Vrtilek, S. D., 2008. The eccentric accretion disc of the black hole A0620-00. MNRAS, 384, 849.

O'Brien, K., Horne, K., Hynes, R. I., Chen, W., Haswell, C. A., Still, M. D., 2002. Echoes in X-ray binaries. MNRAS, 334, 426-434.

Oke, J. B., 1977. Further spectrophotometry of the transient X-ray source A0620-00. ApJ, 217, 181-185.

Oke, J. B., Greenstein, J. L., 1977. Spectrophotometry of the transient X-ray source A0620-00. ApJ, 211, 872-880.

Olson, B. I., 1975. On the ratio of total-to-selective absorption. PASP, 87, 349-351.

Orosz, J. A., Bailyn, C. D., 1997. Optical observations of GRO J165540 in quiescence. I. A precise mass for the black hole primary. ApJ, 477, 876.

Orosz, J. A., Hauschildt, P. H., 2000, The use of the NextGen model atmospheres for cool giants in a light curve synthesis code. A\&A, 364, 265-281.

Orosz, J. A., Jain, R. K., Bailyn, C. D., McClintock, J. E., Remillard, R. A., 1998. Orbital parameters for the soft X-ray transient $4 \mathrm{U}$ 1543-47: Evidence for a black hole. ApJ, 499, 375.

Osterbrock, D., Fulbright, J. P., Martel, A. R., Keane, M. J., Trager, S. C., Basri, G., 1996. Night-Sky High-Resolution Spectral Atlas of $\mathrm{OH}$ and $\mathrm{O} 2$ Emission Lines for Echelle Spectrograph Wavelength Calibration. PASP, 108, 277.

Paczyński, B., 1971. Evolutionary processes in close binary systems. ARA\&A, 9, 183.

Pal, S., Chakrabarti, S. K., Jun 2005. A GHz flare in a quiescent black hole and a determination of the mass accretion rate. Chinese Journal of Astronomy and Astrophysics 5, 331

Phillips, S. N., Shahbaz, T., Podsiadlowski, P., 1999. The outburst radial velocity curve of X-ray Nova Scorpii 1994 (=GRO J165540): a reduced mass for the black hole? MNRAS, 304, 839-844.

Predehl, P., Schmitt, J. H. M. M., Jan 1995. X-raying the interstellar medium: ROSAT observations of dust scattering halos. A\&A, 293, 889.

Radhakrishnan, V., Goss, W. M., Murray, J. D., Brooks, J. W., Jan 1972. The parkes survey of 21-centimeter absorption in discretesource spectra. III. 21-centimeter absorption measurements on 41 galactic sources north of declination -48 degrees. Astrophysical Journal Supplement, 24, 49.

Rees, M. J., 1966. Appearance of relativistically expanding radio sources. Nature, 211, 468.

Regős, E., Tout, C. A., Wickramasinghe, D., 1998. The unusual evolutionary state of GRO J1655-40. ApJ, 509, 362-365.

Remillard, R. A., Muno, M. P., McClintock, J. E., Orosz, J. A., 2002 Evidence for harmonic relationships in the high-frequency quasiperiodic oscillations of XTE J1550-564 and GRO J1655-40. ApJ, 580, 1030-1042.

Sala, G., Greiner, J., Vink, J., Haberl, F., Kendziorra, E., Zhang, X. L., 2007. The highly ionized disk wind of GRO J1655-40. A\&A, 461, 1049-1056.

Sánchez-Fernández, C., Castro-Tirado, A. J., Duerbeck, H. W., Mantegazza, L., Beckmann, V., Burwitz, V., Vanzi, L., Bianchini, A., della Valle, M., Piemonte, A., Dirsch, B., Hook, I., Yan, L., Giménez, A., 1999. Optical observations of the black hole candidate XTE J1550-564 during the september/october 1998 outburst. A\&A, 348, L9-L12.

Schmidt, M., Jan 1965. Rotation parameters and distribution of mass in the galaxy. Galactic structure. Edited by Adriaan Blaauw and Maarten Schmidt, University of Chicago Press, 513.

Shahbaz, T. 2003. Determining the spectroscopic mass ratio in interacting binaries: application to X-Ray Nova Sco 1994, MNRAS,
339,1031

Shahbaz, T., Hynes, R. I., Charles, P. A., Zurita, C., Casares, J., Haswell, C. A., Araujo-Betancor, S., Powell, C., Oct 2004. Optical spectroscopy of flares from the black hole X-ray transient A062000 in quiescence. MNRAS, 354, 31

Shahbaz, T., Naylor, T., Charles, P. A., Jun 1994. The mass of the black hole in A0620-00. MNRAS, 268, 756.

Shahbaz, T., van der Hooft, F., Casares, J., Charles, P. A., van Paradijs, J., 1999. The mass of X-ray nova Scorpii1994 (=GRO J1655-40). MNRAS, 306, 89-94.

Shaposhnikov, N., Titarchuk, L., 2009 . Determination of Black Hole Masses in Galactic Black Hole Binaries using Scaling of Spectral and Variability Characteristics. arXiv:09022852v1.

Shaver, P. A., Radhakrishnan, V., Anantharamaiah, K. R., Retallack, D. S., Wamsteker, W., Danks, A. C., 1982. Anomalous motions of H I clouds. A\&A, 106, 105.

Soria, R., Wu, K., Hunstead, R. W., 2000. Optical spectroscopy of GRO J1655-40. ApJ, 539, 445-462.

Spitzer, L., Sep 1948. The distribution of interstellar sodium. ApJ, $108,276$.

Stebbins, J., Huffer, C. M., Whitford, A. E., Jan 1940. The colors of 1332 B stars. ApJ, 91, 20.

Stevens, J. A., Hannikainen, D. C., Wu, K., Hunstead, R. W., McKay, D. J., 2003. The radio flaring behaviour of GRO J1655-40: an analogy with extragalactic radio sources? MNRAS, 342, 623-628.

Takahashi, H., Fukazawa, Y., Mizuno, T., Hirasawa, A., Kitamoto, S., Sudoh, K., Ogita, T., Kubota, A., Makishima, K., Itoh, T., Parmar, A. N., Ebisawa, K., Naik, S., Dotani, T., Kokubun, M., Ohnuki, K., Takahashi, T., Yaqoob, T., Angelini, L., Ueda, Y., Yamaoka, K., Kotani, T., Kawai, N., Namiki, M., Kohmura, T., Negoro, H., 2008. Low/hard state spectra of GRO J1655-40 observed with Suzaku. Publications of the Astronomical Society of Japan, 60, 69.

Tingay, S. J., Jauncey, D. L., Preston, R. A., Reynolds, J. E., Meier, D. L., Murphy, D. W., Tzioumis, A. K., McKay, D. J., Kesteven, M. J., Lovell, J. E. J., Campbell-Wilson, D., Ellingsen, S. P., Gough, R., Hunstead, R. W., Jones, D. L., McCulloch, P. M., Migenes, V., Quick, J., Sinclair, M. W., Smith, D., 1995. Relativistic motion in a nearby bright X-ray source. Nature, 374, 141.

Trimble, V., Leonard, P. J. T., 1995. Astrophysics in 1994. PASP, 107, $1-21$.

Tsunemi, H., Kitamoto, S., Okamura, S., Roussel-Dupre, D., 1989. Discovery of a bright X-ray nova, GS 2000+25. ApJ, 337, L81L84.

Ueda, Y., Inoue, H., Tanaka, Y., Ebisawa, K., Nagase, F., Kotani, T., Gehrels, N., Jan 1998. Detection of absorption-line features in the X-ray spectra of the galactic superluminal source GRO J1655-40. ApJ, 492, 782.

van der Hooft, F., Groot, P. J., Shahbaz, T., et al. 1997. The black hole transient Nova Scorpii 1994 (=GRO J1655-40): orbital ephemeris and optical light curve, MNRAS, 286, L43

van der Hooft, F., Heemskerk, M. H. M., Alberts, F., van Paradijs, J., 1998. The quiescence optical light curve of Nova Scorpii 1994 (=GRO J1655-40). A\&A, 329, 538-550.

van der Woerd, H., White, N. E., Kahn, S. M., 1989. X-ray spectroscopy of the ultrasoft transient 4U 1543-47. ApJ, 344, 320-324.

Vrtilek, S. D., McClintock, J. E., Seward, F. D., Kahn, S. M., Wargelin, B. J., 1991. The Einstein objective grating spectrometer survey of galactic binary X-ray sources. ApJS, 76, 1127-1167.

Wagner, R. M., Starrfield, S. G., Hjellming, R. M., Howell, S. B., Kreidl, T. J., 1994. ROSAT observations of the black hole candidate V404 Cygni in quiescence. ApJ, 429, L25-L28.

Wampler, E. J., Jun 1966. Scanner observations of $\lambda$ 4430. ApJ, 144, 921.

Welsh, B. Y., Vedder, P. W., Vallerga, J. V., 1990. High-resolution sodium absorption-line observations of the local interstellar 
medium. ApJ, 358, 473.

West, R. M., della Valle, M., Jarvis, B., Pizarro, G., 1991. Nova Muscae 1991 (X-ray transient in Musca). IAU Circular 5165.

Willems, B., Henninger, M., Levin, T., Ivanova, N., Kalogera, V., McGhee, K., Timmes, F. X., Fryer, C. L., 2005. Understanding compact object formation and natal kicks. I. Calculation methods and the case of GRO J1655-40. ApJ, 625, 324-346.

Wu, C.-C., Aalders, J. W. G., van Duinen, R. J., Kester, D., Wesselius, P. R., 1976. A study of the transient X-ray source A0620-00. A\&A, 50, 445-449.

Wu, C.-C., Panek, R. J., Holm, A. V., Schmitz, M., Swank, J. H., 1983. Ultraviolet observations of the transient X-ray sources A0535+26 and A0620-00. PASP, 95, 391-397.

Xiang, J., Lee, J. C., Nowak, M. A., 2007. Using the X-ray dust scattering halo of $4 \mathrm{U} 1624-490$ to determine distance and dust distributions. ApJ, 660, 1309.

Yamaoka, K., Ueda, Y., Inoue, H., Nagase, F., Ebisawa, K., Kotani, T., Tanaka, Y., Zhang, S. N., 2001. Asca observation of the superluminal jet source GRO J1655-40 in the 1997 outburst. PASJ, 53, 179-188.

York, D. G., May 1971. Structure in the interstellar-extinction curve ApJ, 166, 65.

Zhang, S. N., Ebisawa, K., Sunyaev, R., Ueda, Y., Harmon, B. A., Sazonov, S., Fishman, G. J., Inoue, H., Paciesas, W. S., Takahashi, T., 1997. Broadband high-energy observations of the superluminal jet source GRO J1655-40 during an outburst. ApJ, 479, 381.

Zhang, S. N., Harmon, B. A., Paciesas, W. S., Fishman, G. J., 1996a. Deep search for celestial hard X-ray emission by Earth occultation with BATSE/CGRO. A\&AS120, C137+.

Zhang, S. N., Harmon, B. A., Paciesas, W. S., Fishman, G. J., Finger, M. H., Robinson, C. R., Rubin, B. C., Grindlay, J. E., Barret, D., Tavani, M., Kaaret, P., Bloser, P., Ford, E., 1996b. Periodic transient hard X-ray emission from GRO 1849-03. A\&AS120, C227.

Zhang, S. N., Harmon, B. A., Paciesas, W. S., Fishman, G. J., Grindlay, J. E., Barret, D., Tavani, M., Kaaret, P., Bloser, P., Ford, E., Titarchuk, L., 1996c. Low state hard X-ray outburst from the Xray burster 4U 1608-522 observed by BATSE/CGRO. A\&AS120, C279.

Zhang, S. N., Wilson, C. A., Harmon, B. A., Fishman, G. J., Wilson, R. B., Paciesas, W. S., Scott, M., Rubin, B. C., 1994. X-ray nova in scorpius. IAU Circular 6046, 1. 